\title{
Multiorgan Motion Tracking in Dynamic Magnetic Resonance Imaging for Evaluation of Pelvic System Mobility and Shear Strain
}

\author{
Z. Jiang $2,6,7$, J.-F. Witz ${ }^{1,7}$, P. Lecomte-Grosbras ${ }^{1,7}$, J. Dequidt ${ }^{2,6}$, S. Cotin ${ }^{5}$, C. Rubod ${ }^{1,3,4}$, C. \\ Duriez $^{5}$ and M. Brieu ${ }^{1,7}$ \\ ${ }^{1}$ FRE 3723 - LML - Laboratoire de Mcanique de Lille, Univ. Lille, F-59000 Lille, France \\ ${ }^{2}$ Univ. Lille, UMR 9189 - CRIStAL - Centre de Recherche en Informatique Signal et Automatique de Lille, F-59000 Lille, France \\ ${ }^{3} \mathrm{CHU}$ Lille, Gynaecology Department, Hôpital Jeanne de Flandre, F-59000 Lille, France \\ ${ }^{4}$ Faculty of medicine, Lille University, F-59000 Lille, France \\ ${ }^{5}$ Inria, University of Lille, France \\ ${ }^{6}$ CNRS, UMR 9189, F-59000 Lille, France \\ ${ }^{7}$ Centrale Lille, F-59000 Lille, France
}

\begin{abstract}
Female pelvic disorders have a large social impact, the diagnosis of which relies on a key indication: pelvic mobility. The normal mobility is present in a healthy patient, meanwhile the hypermobility can be a sign of female pelvic prolapse and the hypomobility for endometriosis. The evaluation of pelvic mobility is based on medical image analysis. However, the latter does not provide precise values of these indicators directly. Moreover, suspension devices play an important role in pelvic organ function, but can hardly be observed on medical images. Our objective is to propose an image-based analysis tool for the quantitative evaluation of pelvic mobility and the shear strain which has an impact on suspension devices. Hence, this paper introduces a such tool based on an efficient and semi-automatic motion tracking of multiple pelvic organs: the bladder, vagina and rectum presented in dynamic magnetic resonance imaging sequences. The method was validated on prototypical images and applied to different mobility cases. The computed displacement and shear strain fields provide important information on the quality of suspension devices between organs for a fine diagnosis in the clinical context, for example the early diagnosis of female pelvic prolapse and the localization of possible lesion areas before surgery. Meanwhile, the predicted mobility can be used to compare with the finite element model for numerical simulation.
\end{abstract}

KEY WORDS: displacement field, image correlation, motion tracking, MRI, pelvic system mobility, shear strain

\section{Introduction}

The background knowledge about female pelvic disorders has shown that the incidence of both genital prolapse and pelvic endometriosis is relatively high. More precisely, the prolapse affects 20 to $30 \%$ of women of all ages combined and $60 \%$ of women over 60 years old $[1,2]$. The endometriosis affects about $10 \%$ of women in general [3], and its incidence is much higher in infertile women, which can reach 
$47 \%$ [4]. These problems are strongly indicated by the pelvic hypermobility [5] and hypomobility [6]. In order to analyse the pelvic mobility, LecomteGrosbras et al. [7] have recently proved the interest, necessity and feasibility for involving numerical tools (full-field measurement based on medical images) in the medical diagnosis and treatment of pelvic pathologies. The conventional diagnosis relies on the human interpretation of dynamic MR images [8]. Such analysis drives to subjectivity to a certain degree and is limited due to some hardly observable structures on MR images such as ligaments and fascias. However, as mentioned in [7], the technique of Image Registration (IR) or Digital Image Correlation (DIC) can provide more objective and complete information on the pelvic mobility in a in vivo manner. This information enables a finer diagnosis and would help to predict some possible lesion areas before surgery. Moreover, it can provide useful data for validation of the finite element (FE) simulation. The latter aims to have a better understanding of the mechanism of pelvic system dysfunction $[9,10,11]$.

Whereas the technique of full-field measurement based on IR and DIC has become mature in mechanical experiments and its application in medical images has been widely developed [12, 13, 14, 15], the issue of its limitations and inconveniences in our study should still be addressed. Firstly, in the case of ex vivo testing, one can create a random pattern on the image by painting the object with speckles, which enables to identify the material points [16]. However, in our in vivo case, such procedure can not be reproduced. Hence, it would be difficult to take account of the tracking of material points. Although the Tagging Dynamic MRI has been existing for motion analysis on some particular organs such as the heart and the tongue $[17,18]$, the tagging is not performed in our current imaging protocol and we want to adapt our analysis tool to clinical use without asking for new acquisition protocol. Secondly, the full-field technique provides promising results on the whole image domain. However, we need information especially on the organ walls (on the contours in 2D manner) and on the interface between the organs (vesico-vaginal space and recto-vaginal space). So far masked maps have been created manually over time in the image sequence to extract the information on the contours. This manual work is too tedious to be applied in routine to patient-specific cases. Finally, the suspension devices are difficultly observed on dynamic MR images. Such interesting relative information between the organs cannot be obtained by conventional registration methods. Precisely, the information to be computed includes the displacements field on the organ walls and the shear strain on the interface between the organs. The shear strain can not be measured and is to be computed numerically by considering the middle organ, the vagina, as the reference. It provides a model of the behavior of some structures such as ligaments and fascias, as mentioned, hardly observable in the images. Whereas, the damage of fascias causes the hypermobility.

Our objective is to introduce an image-based analysis tool for the evaluation of pelvic mobility that takes account of the aforementioned limitations. The method enables the evaluation of the displacement and the shear strain over time and in the zone of interest.

In [19], we proposed a registration method for multi-organ detection in MR images based on Bspline models. Firstly, we extended the detection method on an image to a sequence of dynamic MR images for multi-organ tracking over time. The Bspline model is initialized on the first image (instant $t_{0}$ ) by the original method. Then the method is used iteratively to deform the model from instant $t$ to $t+1$ in the rest of the sequence: the fitted model at instant $t$ becomes the initial model at instant $t+1$. The number of control points remains constant to compute the displacement field in a same configuration.

Nevertheless, the major concern is to ensure the plausibility of the predicted deformation of the organs, the evaluation of the displacement and the shear strain over time. It is not ensured that the material points are well followed and the points cannot drift along the contour inducing a non realistic movement. In fact, as mentioned before, this sliding issue has been wildly known for image correlation problems [20]. For this reason, another strategy is 
required to ensure the best possible tracking of material points.

In this paper, we propose a new numerical approach which enables not only the evaluation of pelvic mobility, but also the shear strain between organs. A geometric model of composite Bézier contours with some identifiable material points is used. They are initialized on the first image in the dynamic sequence. These contours are then deformed and followed fully automatically over time to fit the organ shapes in each image of the sequence. Finally, using this analytical model, we can compute displacement fields on the contours of organs to quantify the motion and shear strains inter-organs that are induced by the biomechanical properties of structures.

This approach brings several contributions: (1) Parametric and analytical model is used for the description of the contours of organs. The tracking of the contours is performed on its control points and is fully automatic. Hence, the application to patient-specific cases can also be fully considered. (2) The automatic detection method based on Bspline is used for the initialization [19]. However, in order to predict more physically meaningful displacements, several material points are taken into consideration, based on the criteria of curvature. (3) The displacement field and shear strain are obtained and computed directly by using its analytical formulation.

For the reason of clarity, the extended B-spline based method [19] and the new Bézier based method in this paper are denoted as method "Geometric contours tracking" and "Material points tracking", respectively, in the remainder of this paper. In the rest of the paper, we present the motion tracking algorithm without material points (B-spline) and with material points (Bézier). Their difference is compared and illustrated by several tests with prototypical and medical images. Finally, the application of the method is performed on a set of data in different contexts of mobility.

\section{Magnetic Resonance Imaging (MRI) Data}

Thanks to its high contrast for soft tissues and without the exposure to radiative sources such as X-ray, magnetic resonance imaging has become the firstchoice technique in clinical examinations on female pelvic system [21]. Particularly in our study, dynamic MRI (or cine-MRI) is used for the evaluation of pelvic mobility. This type of MRI is widely performed in clinical routine for the diagnosis of pelvic diseases such as prolapse [8]. The images consist of a temporal sequence of MRI recording the movements of pelvic system while the patient is pushing down. The experimental data of healthy volunteers dedicated to research were used and the clinical data of patients with pathologies were used under the approval by the National Ethics Committee (Comité d'Ethique de la Recherche en Obstétrique et Gynécologie CEROG 2012-GYN-06-01-R1).

Figure 1 shows an example of the dynamic MRI of a healthy witness patient. In order to highlight the organs and their mobility, intravaginal and intrarectal gel had been introduced into the two organs, and the bladder was semi-replete. These images are $T_{1}$-weighted, the spatial resolution of which is about $1.17 \mathrm{~mm} \mathrm{pixel}^{-1}$ and the temporal resolution is about 1 image per second. The MRI acquisition protocol is detailed as follows: magnetic field strength $1.5 \mathrm{~T}$; repetition time $2.7 \mathrm{~ms}$; echo time 1.35 ms; slice thickness $10 \mathrm{~mm}$; pixel bandwidth $1225 \mathrm{~Hz}$; flip angle: $45^{\circ}$.

Theoretically the dynamic MRI can be acquired in $3 \mathrm{D}$ as the static ones, yet the choice of a fixed plane for the dynamic MRI acquisition has become a standard practical protocol in the clinical routine. Because the aim of the analysis tool is to provide practical applications in diagnoses, we have to respect the image modality in clinical use, for example no specific requirement of higher resolution has been demanded. Meanwhile the logistical, budget and time constraints are also respected. For these reasons, we have possession of $2 \mathrm{D}$ dynamic MRI in sagittal median plane which is the most representative one for highlighting the motions. One of the limitations due to the $2 \mathrm{D}$ image modality is that 


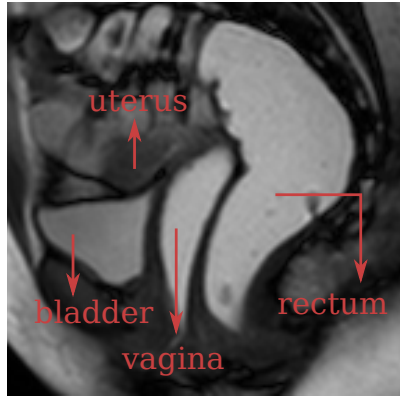

Initial image

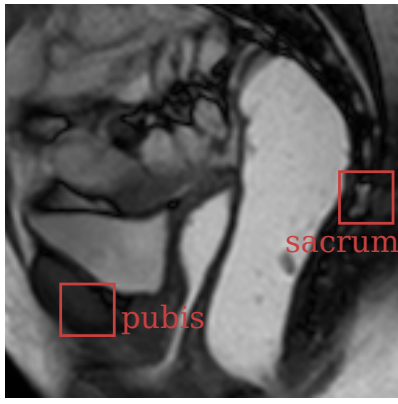

Deformed image
Figure 1: Dynamic magnetic resonance image of a healthy witness patient (median sagittal plane, $T_{1}$-weighted, spatial resolution $=1.17 \mathrm{~mm}$ pixel ${ }^{-1}, 256 * 256$ pixels).

one component of the displacements cannot be observed. In the diagnosis, it is considered that the displacements in this missing direction are sufficiently small to not necessitate a 3D image modality. Consequently, in our approach, we analyse only the $2 \mathrm{D}$ motion in the sagittal plane.

\section{B-spline based Multi-organ Detection in dynamic MRI}

In order to assess the movement of organs involved in the genital prolapse, pelvic endometriosis or the normal context, we have to build the initial models of bladder, vagina and rectum on the first image, as the reference configuration. These models are defined as adaptive cubic B-splines, which consist of closed contours in our case, even for the vagina and rectum which have in reality open ends. Then these models are deformed iteratively on the following images in the sequence. In [19] we had presented a model-to-image registration approach for detecting multiple organs and building these B-spline models in MRI. We are to apply this method for the Bspline based tracking. Thus, we will describe briefly its principle by listing several formulas and notations which will be used in this paper. For further details, the reader can refer to the aforementioned paper.

Each organ contour is defined by using a B-spline curve. A uniform knot vector $[0 \ldots 1]$ is used to compute spline functions of degree $p, \mathcal{N}_{i, p}:[0,1] \rightarrow[0,1]$ (definition can be found in [22]). The organ is then presented by a parametric curve $\mathcal{B}: u \in[0,1] \rightarrow$ $\mathcal{B}(u) \in \mathbb{R}^{2}$. The position of a point in the neighbourhood of the B-spline can be computed with the parametric coordinate $u$ and the distance between the point and the curve $v$. Let $\mathbf{n}(u)$ be the vector normal to the curve $\left(\mathbf{n}(u) \in \mathbb{R}^{2}\right)$, the position $\mathbf{x}(u, v)$ can be given by:

$$
\begin{aligned}
\mathbf{x}(u, v) & =\mathcal{B}(u)+v \mathbf{n}(u) \\
& =\sum_{i=0}^{n} \mathcal{N}_{i, p}(u) \mathbf{p}_{i}+v \mathbf{n}(u),
\end{aligned}
$$

where $\left[\mathbf{p}_{0}, \mathbf{p}_{1}, \cdots \mathbf{p}_{n}\right]$ are the $n+1$ control points. Obviously $\mathbf{x}(u, 0)$ correspond to a point on the Bspline curve.

The registration method aims to optimize the $n+1$ control points by minimizing an objective function having these points as degrees of freedom. This function measures the difference between the real image (MRI) and the virtual one which is defined in the neighbourhood of the contour $\Omega$ by a so-called levelset function. The levelset function defines the value of grey levels in the virtual image which is similar to the ones in the real image. The use of virtual image was introduced in [23] and discussed in $[24,25]$. Now let $\tau$ be the neighbourhood width. The levelset $\mathcal{L}$ is given by the following formula:

$$
\mathcal{L}(v)=\left\{\begin{array}{l}
A_{\text {max }} \quad \text { if } \quad v<0, \\
A_{\text {min }}+\frac{A_{\text {max }}-A_{\min }}{2} *\left(1+\cos \left(\frac{\pi v}{\tau}\right)\right) \\
\quad \text { if } \quad 0 \leq v \leq \tau, \\
A_{\min } \quad \text { if } \quad v>\tau,
\end{array}\right.
$$

where $A_{\max }$ is the grey level inside of the organ and $A_{\min }$ the background. The values of $A_{\min }$ and $A_{\max }$ are chosen by clicking inside and outside of each organ in the real image. As for the width $\tau$, it measures the width of the transition zone between the organ and the background. In fact, sharp lines separating these two parts do not exist in the real image. Hence, the choice of its value depends actually on the desired position of the final contour 
(closer to the foreground or to the background). In our application we have chosen a standard and fixed value ( 3 pixels). Thus, the grey level value on a position $\mathbf{x}$ of the virtual image $I_{v}$ can be computed analytically:

$$
I_{v}(\mathbf{x}(u, v))=\mathcal{L}(v) .
$$

Finally, the objective function $E_{\text {image }}$ measures the squared difference between the real image $I_{r}$ and the virtual one $I_{v}$.

$$
\begin{aligned}
& E_{\text {image }}\left(\left[\mathbf{p}_{0}, \mathbf{p}_{1}, \cdots \mathbf{p}_{n}\right]\right)=\sum_{\mathbf{x} \in \Omega}\left[I_{r}(\mathbf{x})-I_{v}(\mathbf{x})\right]^{2} \\
& =\sum_{u} \sum_{v}\left[I_{r}\left(\mathcal{B}\left(u ; \mathbf{p}_{0}, \mathbf{p}_{1}, \cdots \mathbf{p}_{n}\right)+v \mathbf{n}(u)\right)-\mathcal{L}(v)\right]^{2} .
\end{aligned}
$$

The minimization of this function enables to find the optimums of control points, $i$. e. the best correlation between the B-spline model and the MRI image. Hence, the optimized B-spline gives the initial configuration of the tracking problem. Then, these control points are to be optimized iteratively by using the same minimization formulation. Besides the geometric contour, the material points have to be taken into account. In the next section, the new method "Material points tracking" based on Bézier models will be presented.

\section{Motion Tracking Algorithm}

\section{Tracking principle}

In our approach, based on continuum mechanics, we use a Lagrangian description to define the motions of the principal pelvic organs under deformation. Following this description, for any material point of the object, the change of its position over the time is defined by a function of transformation, from its initial configuration (or reference) to the current configuration. In other words, at any time, the computation of physical quantities such as the displacement is always associated with the same material point, compared to its initial configuration.

To achieve the objective of motion tracking, firstly, we initialize the B-spline geometric model at instant $t_{0}$ (the first image of the sequence) as the reference configuration by using the model-to-image registration [19], as described in Section "B-spline based Multi-organ Detection in dynamic MRI". Secondly, the model is updated for fitting each following image in the sequence to form the configuration at instant $t$. The process is recursive: the model of instant $t$ is deformed to fit the next image at instant $t+1$, and it is also based on an energy minimization formulation. Once we obtain the models corresponding to all the instants, a displacement and shear field can be computed by using the analytical formulation of the models and following the Lagrangian description.

Meanwhile, we need to deal with the major concern about the plausibility of computed displacements. Normally, as mentioned in the previous paragraph, the description of the movement of material points remains in a fix landmark related to the initial configuration. So far the computation is based on the parametric coordinates, depending on the control points and their relative shift. Unfortunately these control points are not located on contours and they can freely drift along the contour. Thus the landmark for calculating the position of material points is mobile.

The idea is to fix some points on corners and to restrict the movement to be perpendicular to the contour. For this purpose, the B-spline model is divided into piecewise cubic Bézier curves joined end to end. The ending point of one Bézier curve is attached with the starting point of another to form a connection point which is considered as a material point and where the curvature is higher than otherwhere. Here make we an anatomical assumption that the salient points on the organ surface (or corners on the contour in a $2 \mathrm{D}$ plan) represent material points, which is based on the observation of medical images. Such material points, denoted as "features" in the remainder of this paper, are firstly tracked by the optical flow algorithm [26], which consist in the beginning configuration for each recursive process of deformable registration from $t$ to $t+1$. Figure 2 shows the principal pipeline of the motion tracking.

In the following parts, we will present respectively 


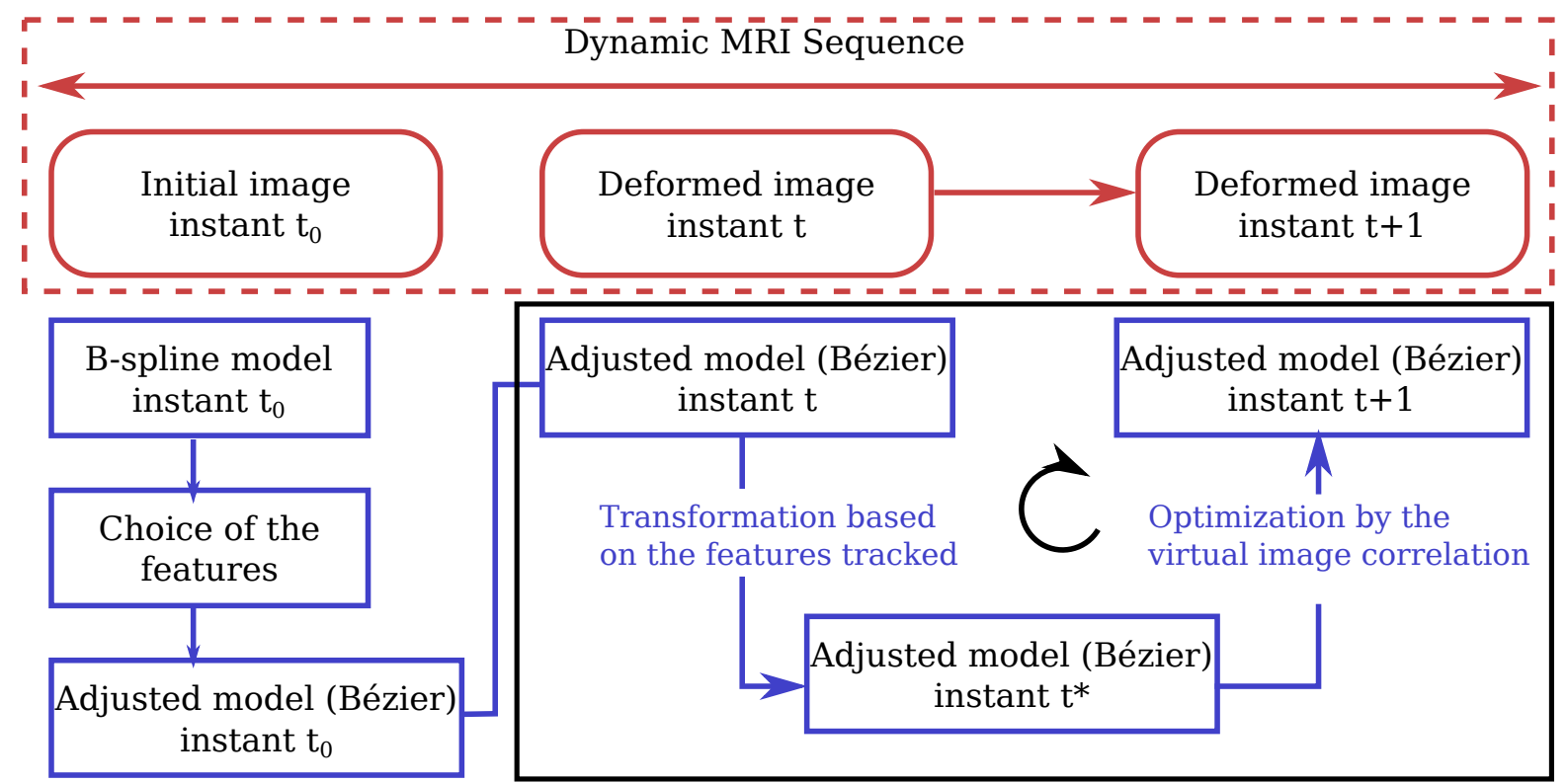

Figure 2: Motion tracking principle: the initialization of the contours on the first image is carried out by the B-spline based virtual image correlation $[23,25,19]$. Then the features are selected automatically based on curvatures. The B-spline model contour is divided into piecewise Bézier curves (called the adjusted model) to obtain the initial model corresponding with the image at instant $t_{0}$. For the other images in the sequence, the features and the Bézier curve are deformed in two steps.

the choice of the features, the motion tracking algorithm, the computation of displacement and shear field.

\section{Choice of the features}

We apply a new adjusted model (composite Bézier curve) and the connection points of Bézier curves become the material points. At the initial step, the choice of these features is performed automatically based on detection of the higher curvatures.

Suppose that the contour of an organ in the initial configuration (instant $t_{0}$ ) is defined by a parametric B-spline curve $\mathcal{B}^{0}(u)=\sum_{i=0}^{n-1} \mathcal{N}_{i, 3}(u) \mathbf{p}_{i}$. The absolute value of the curvature is defined as the rate of change of the unit tangent vector. In a parametric formulation, it is related to the first and second derivative of the parametric function, which can be calculated by using the standard parametric coordi- nates as follows:

$$
\kappa(u)=\frac{\left\|\mathcal{B}^{0^{\prime}}(u) \times \mathcal{B}^{0^{\prime \prime}}(u)\right\|}{\left\|\mathcal{B}^{0^{\prime}}(u)\right\|^{3}},
$$

where $\mathcal{B}^{0^{\prime}}(u)=\frac{\mathrm{d} \mathcal{B}^{0}}{\mathrm{~d} u}(u), \mathcal{B}^{0^{\prime \prime}}(u)=\frac{\mathrm{d}^{2} \mathcal{B}^{0}}{\mathrm{~d} u^{2}}(u)$ and $\times$ for the cross product. Hence, the curvilinear curvature can be computed directly based on the initial B-spline model. In an automatic way, the algorithm enables to select a number of features where the curvature is higher by using the following criteria:

- maximal and minimal number of features to be selected;

- minimum distance between two adjacent features (used in order that the features are well spaced);

- threshold of the curvature (used to avoid the features on flat segments). 
Figure 3 shows the selected features with a curvature map bounding the contour. Meanwhile, the features are resorted (renumbered) counter clockwise along the B-spline.

Let $\left[\mathbf{p f}_{0}, \mathbf{p f}_{1}, \cdots, \mathbf{p f}_{l}\right]$ be the selected feature points, where $\mathbf{p} \mathbf{f}_{i}=\mathcal{B}^{0}\left(u_{k_{i}}\right) . l+1$ piecewise Bézier curves can be created and joined at these points, which represent the approximative geometry as the initial B-spline and constitute the adjusted model. However, the continuity at the connecting points is reduced. As defined in many standard texts [22], the Bézier curve is a special case of the B-spline who has four control points. In this case, for each piecewise Bézier curve, the two extremities are fixed by two adjacent features. Let us take the first Bézier segment between $\mathbf{p f}_{0}$ and $\mathbf{p f _ { 1 }}$ as an example. This curve, denoted $\mathcal{S}_{0}$, is defined by Equation 7 with the Bernstein polynomial $\mathcal{M}_{i, p}: u \in[0,1] \rightarrow[0,1]$ :

$$
\begin{gathered}
\mathcal{M}_{i, p}(u)=\frac{p !}{i !(p-i) !} u^{i}(1-u)^{p-i}, \\
\mathcal{S}_{0}(u)=\sum_{i=0}^{3} \mathcal{M}_{i, 3}(u) \mathbf{p}_{i},
\end{gathered}
$$

where $\mathbf{p}_{0}=\mathbf{p f}_{0}$ and $\mathbf{p}_{3}=\mathbf{p f}_{1}$. Then the two free control points $\mathbf{p}_{1}$ and $\mathbf{p}_{2}$ are determined such that each Bézier curve fits the original B-spline contour on the interval $\left[u_{k_{0}}, u_{k_{1}}\right]$. The curve fitting method is well known as a standard approach by solving a system of linear equations. Figure 4 shows the adjusted Bézier model as the initial configuration, created from the original B-spline model. In further studies, we will use this well-defined adjusted model for analysing organ movements.

\section{Tracking of features}

In order to ensure the quality of the computation of mechanical indicators, such as the displacements and shear strain, several constraints have been included not only for the detection of geometric contour, but also for the better prediction of the motion of organs under deformation. Moreover, we are interested in the organ walls not their inside for analysing the pelvic mobility. Hence, in the first time, the tracking of motion should be guided by the features on the contour.

For each piecewise Bézier curve, the positions of the two end control points (material points) and the free ones are optimized in different ways. In this section, the tracking of the two end points will be firstly discussed. This problem can be formulated as detecting moving characteristic points in a video or image sequence. The approach of optical flow proposed by Lucas et al. [26] can be well adapted into our needs. The method is based on the assumption of the constant pixel intensities and their low local variation. Because of the local evaluation, the method would fail if the deformation between images was large or the intensity in a region was uniform [27]. However, in our case, the features to be tracked are located on the edge of organs where the variation of pixel intensities is large. Moreover, with the acquisition frequency of about one image per second, the deformation between two images (instant $t$ and $t+1)$ is relatively small. All these aspects permit a good performance of the optical flow tracking. In a practical view, it is necessary to choose the appropriate window grid size for the computation which respects the properties of locality and where the image pattern is enough representative to be tracked. Generally, the choice of size depends on the image, we have adjusted this parameter empirically during tests (see Figure 5 for illustration of results).

Once the tracking of features is completed using the optical flow, the transformation of each feature has to be applied over its two adjacent free control points to form the intermediate model at instant $t^{*}$ (between $t$ and $t+1$ ). Let always $\mathcal{S}_{0}: u \in[0,1] \rightarrow$ $\mathcal{S}_{0}(u) \in \mathbb{R}^{2}$ be the first Bézier curve with four control points $\left[\mathbf{p}_{0}=\mathbf{p f}_{0}, \mathbf{p}_{1}, \mathbf{p}_{2}, \mathbf{p}_{3}=\mathbf{p f}_{1}\right]$. After applying the transformation, we obtain the following formula:

$$
\begin{aligned}
\mathcal{S}_{0}^{*}(u) & =\mathcal{M}_{0,3}(u) T_{0}\left(\mathbf{p}_{0}\right)+\mathcal{M}_{1,3}(u) T_{0}\left(\mathbf{p}_{1}\right) \\
& +\mathcal{M}_{2,3}(u) T_{1}\left(\mathbf{p}_{2}\right)+\mathcal{M}_{3,3}(u) T_{1}\left(\mathbf{p}_{3}\right),
\end{aligned}
$$

where $T_{0}\left(\mathbf{p f}_{0}\right)=\mathbf{p} \mathbf{f}_{0}^{*}$ and $T_{1}\left(\mathbf{p f}_{1}\right)=\mathbf{p f}_{1}^{*}$ determined by the optical flow method (see Figure 6 for illustrations). 


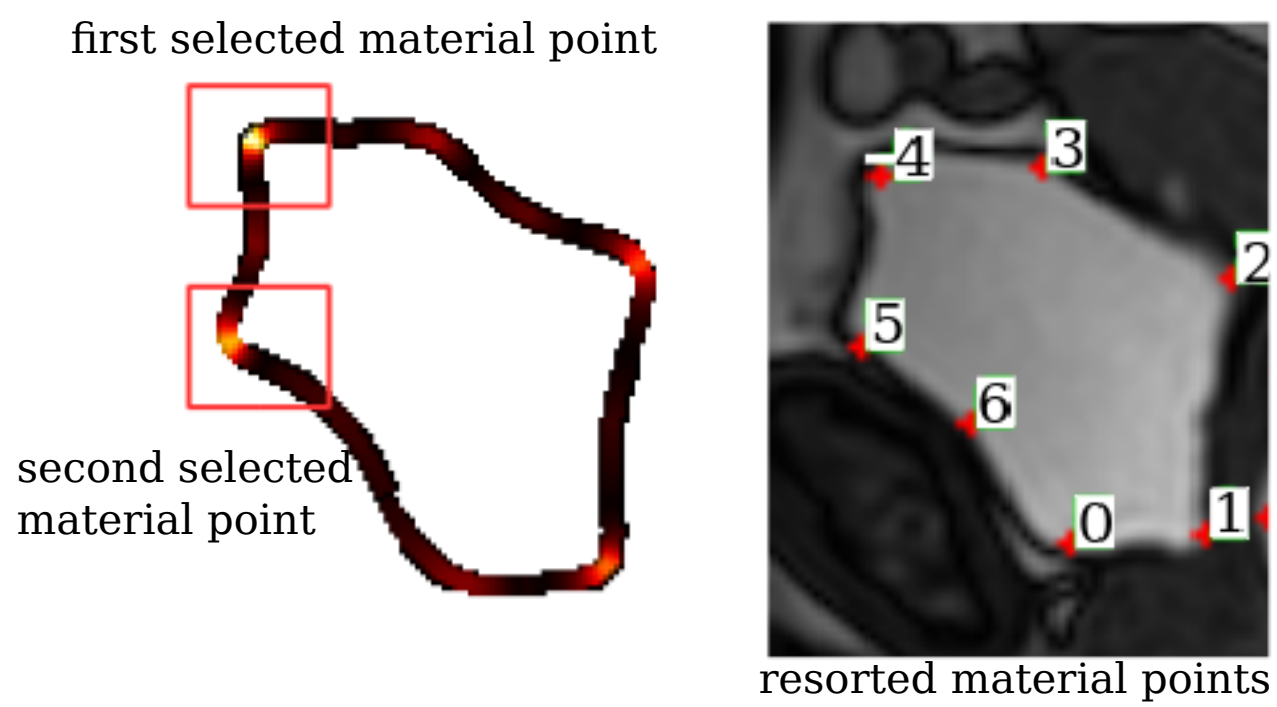

Figure 3: Choice of the features along the B-spline contour: MinDetectRadius means the imposed minimum distance between two adjacent features.

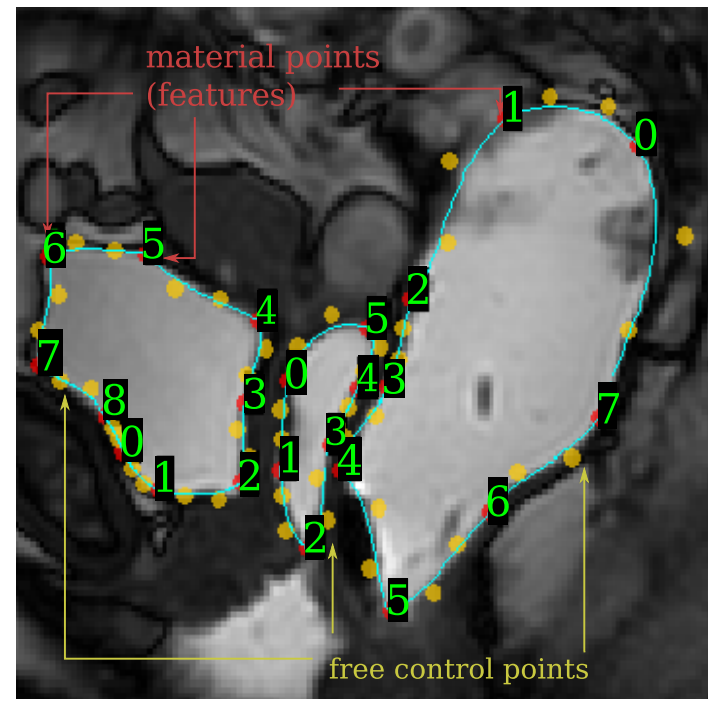

Figure 4: Adjusted model at instant $t_{0}$. The figure is generated by our application (spatial resolution $=1.17 \mathrm{~mm} \mathrm{pixel}^{-1}$ ).

\section{Optimization using Virtual Image Correlation}

As illustrated in Figure 6, the last step consists in adjusting the free control points associated with each Bézier curve so that the model fits perfectly the organ in images. In order to minimize the drifting along the contour, the displacement (transfor-

(C) 2017 The Authors - Journal: Strain 
Initial instant

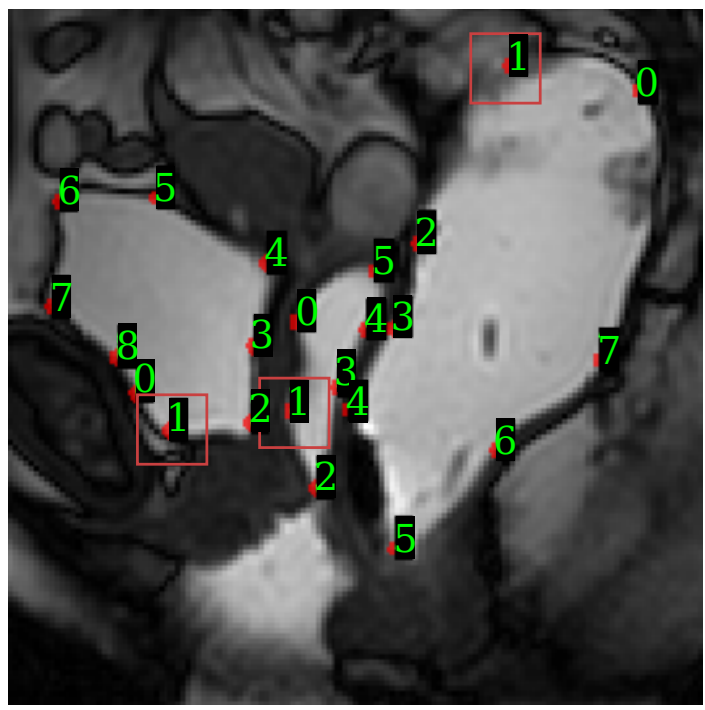

Final instant

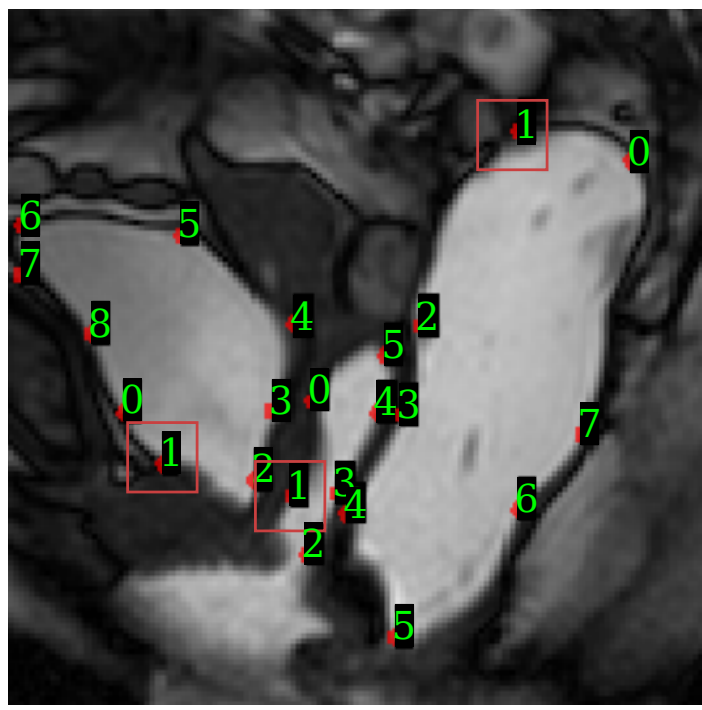

Figure 5: Tracking of the features using the optical flow method: window grid size $=30 \times 30$ pixel $^{2}$.

Instant $t$

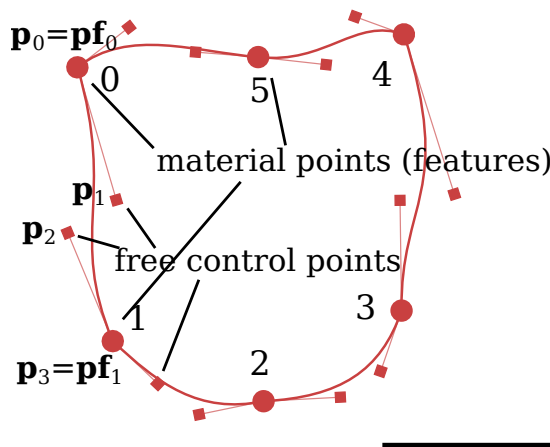

Transformation of the features
Instant $t^{*}$

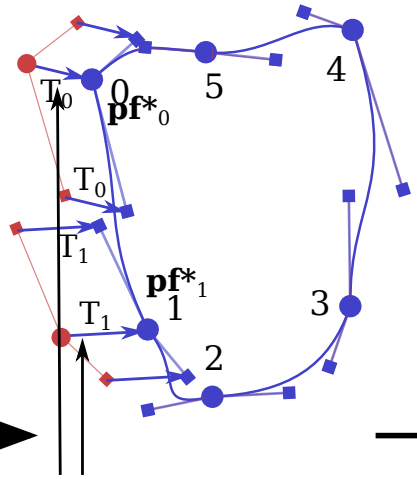

Adjustement of the free control points

Figure 6: Deformation of the model from instant $t$ to $t+1$ : firstly, the features are deformed using optical flow tracking method, for example the transformations of point 0 and 1 are computed by the optical flow equation. Then, the same transformations are applied on their neighbouring free control points to form the intermediate model (instant $t^{*}$ ). Finally, the free control points (for example the ones situated between 0 et 1 ) are adjusted in the direction perpendicular to the segment using virtual image correlation, and produce the model at instant $t+1$. 
cent features. The magnitude of the displacement is also limited to be small because physically no new salient points will be observed during the deformation. Moreover, after the step of transformation based on the optical flow, the intermediate model has been already close to the deformed organ in the image. By examining the width of the transition zone between the organ and the background, we have applied a magnitude of 14 pixels $(-7$ to +7 pixels), which is empirical. In fact, it depends on the resolution of MR images, more precisely, the number of pixels on the contours of the organs. In our cases, the images have the similar resolution. The adjustment allows a finer fit between the model and the image at instant $t+1$. Following the same principle as the B-spline based detection, the adjustment of positions is carried out by minimizing the difference between the virtual image and the real one.

In order to show the computation of the objective function, now let us take the example of the first Bézier curve $\mathcal{S}_{0}$ with control points $\left[\mathbf{p}_{0}=\right.$ $\left.\mathbf{p f}_{0}, \mathbf{p}_{1}, \mathbf{p}_{2}, \mathbf{p}_{3}=\mathbf{p f}_{1}\right]$ as the other free control points are optimized in the same way. Let $\mathbf{N}$ be the normal direction to the segment $\left(\mathbf{p}_{0} \mathbf{p}_{3} \cdot \mathbf{N}=0\right)$. The displacements of the free control points $\mathbf{p}_{1}$ and $\mathbf{p}_{2}$ from their current positions are given by $d_{1}$ and $d_{2}$. The Bézier curve $\mathcal{S}_{0}$ is then redefined by $d_{1}$ and $d_{2}$ :

$$
\begin{aligned}
\mathcal{S}_{0}\left(d_{1}, d_{2} ; u\right) & =\mathcal{M}_{0,3}(u) \mathbf{p}_{0}+\mathcal{M}_{1,3}(u)\left(\mathbf{p}_{1}+d_{1} \mathbf{N}\right) \\
& +\mathcal{M}_{2,3}(u)\left(\mathbf{p}_{2}+d_{2} \mathbf{N}\right)+\mathcal{M}_{3,3}(u) \mathbf{p}_{3} .
\end{aligned}
$$

Analogously to Equation 4, the objective function can be reformulated with respect to $d_{1}$ and $d_{2}$ :

$$
\begin{aligned}
& E_{\text {image }}\left(d_{1}, d_{2}\right) \\
& =\sum_{u} \sum_{v}\left[I_{r}\left(\mathcal{S}_{0}\left(d_{1}, d_{2} ; u\right)+v \mathbf{n}(u)\right)-\mathcal{L}(v)\right]^{2},
\end{aligned}
$$

where $\mathbf{n}(u)$ is the normal direction of the Bézier curve and $\mathcal{L}$ is the levelset function (Equation 2) for computing the virtual image. Then, the function is minimized for each Bézier curve respectively $\left(\mathcal{S}_{0}, \mathcal{S}_{1}, \cdots, \mathcal{S}_{l}\right)$ to obtain the final model, the configuration at instant $t+1$ (Figure 6 ). So far, the optimization is carried out by evaluating the objective function within a restricted range of movements of the two control points of each Bézier segment. From this analytical model, we will be able to compute the displacement and shear field in the zone of interests, which will be presented in the next section.

\section{Displacements and Shears}

The Lagrangian description that we uses enables to compute continuous displacement fields with our analytical geometric models. The displacement fields of each organ must be evaluated separately and each organ must have a reference configuration. This reference corresponds to the shape of the organ wall found at instant $t_{0}$ (on the first image, detected by our registration method). However, practically a given number of discrete points are sampled on the organ contours in the initial configuration. These points are used to compute the displacements field on the organ walls. The analysis of mobility will rely on this discrete domain. The computation of displacements and shears is then based on the parametric coordinates of Bézier curve (see Figure 7A for illustration).

Let $\mathcal{S}_{0}^{0}$ be the first piecewise Bézier curve at instant $t_{0}$. With no loss of generality, $N$ points $\left\{\mathbf{x}_{i}^{0}\right\}$ are sampled on $\mathcal{S}_{0}^{0}$ using the formula:

$$
\mathbf{x}_{i}^{0}=\mathcal{S}_{0}^{0}(i / N), i=0, \cdots, N-1 .
$$

Now, let $\mathcal{S}_{0}^{t}$ be the same Bézier curve at instant $t$. For the set of points, the displacements are given by:

$$
\mathbf{d} \mathbf{p}_{i}^{t}=\mathbf{x}_{i}^{t}-\mathbf{x}_{i}^{0}=\mathcal{S}_{0}^{t}\left(u_{i}\right)-\mathcal{S}_{0}^{0}\left(u_{i}\right) .
$$

After computing the displacements for each Bézier curve respectively, we obtain a set of vectors $\mathbf{d p}_{i}^{t}$ which defines the displacements field at instant $t$, and this computation is repeated for each instant (image) of the dynamic MRI sequence.

Thanks to this registered displacement field, we propose to compute an estimation of the shear strain between organs. The organ in the center of the image, the vagina, is chosen to be the reference organ. Analogously to the computation of displacements, at the initial configuration we generate several couples of discrete points from different organs based on 
A

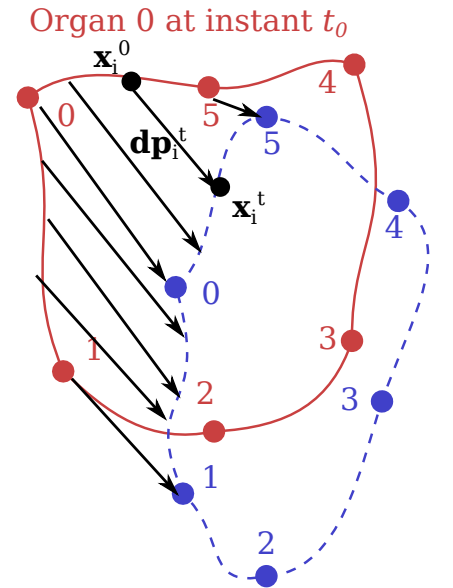

Organ 0 at instant $t$
B Organ 0 at instant $t_{0}$

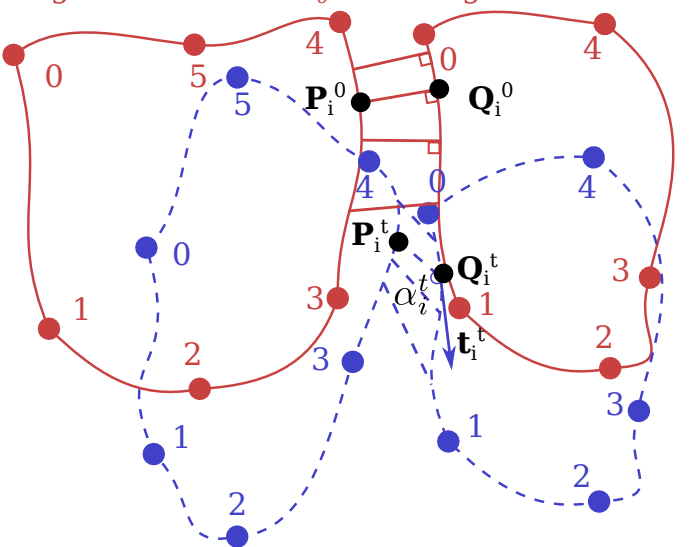

Organ 0 at instant $t$

Organ 1 at instant $t$

Figure 7: Computation of the displacements (A) and shear strain fields (B), between instant $t_{0}$ and $t$.

the zone of interest defined by users. These couples of points are linked by segments, whose direction is perpendicular to the vaginal wall. For example, firstly a part of the bladder contour is selected manually as the zone of interests for computing the shear between the bladder and the vaginal. Then, $M$ discrete points $\left\{\mathbf{P}_{i}^{0}\right\}$ are sampled on this zone. Assuming that the shear deformation is initially null, the points sampled on the bladder are projected orthogonally onto the contour of vagina to obtain $M$ points $\left\{\mathbf{Q}_{i}^{0}\right\}$ who form segments with $\left\{\mathbf{P}_{i}^{0}\right\}$.

Then, in the presence of shear motion, the segments that are all, at the initial stage, normal to the vaginal wall will deviate from this direction and illustrate the shear effect. We use the variation of angles between the segments considered at $t_{0}$ and $t$ as a quantitative measure of the shear strain between organs. The positions of $\mathbf{P}_{i}^{t}$ and $\mathbf{Q}_{i}^{t}$ can be calculated by their displacements $\mathbf{d p}^{t}$. Let $\mathbf{t}_{i}^{t}$ be the tangent vector of vagina at the point $\mathbf{Q}_{i}^{t}$ at instant $t$. The shear strain at the position $\alpha_{i}$ is given by:

$$
\begin{gathered}
\alpha_{i}^{t}=\arccos \left(\frac{\mathbf{Q}_{i}^{t} \mathbf{P}_{i}^{t} \cdot \mathbf{t}_{i}^{t}}{\left\|\mathbf{Q}_{i}^{t} \mathbf{P}_{i}^{t}\right\| \cdot\left\|\mathbf{t}_{i}^{t}\right\|}\right), \\
\operatorname{shear}_{i}^{t}=\tan \left(\frac{\pi}{2}-\alpha_{i}^{t}\right) .
\end{gathered}
$$

Finally, after computing this estimation of the shear strain for each position in the discrete domain, we obtain the set of the scalar values representing the shear strain field at instant $t$ (see Figure 7B for illustration). Moreover, combining the spatial distribution of shear strain over time (the dynamic MRI sequence), we will be able to complete a spatiotemporal representation of the shear between organs which is useful for the analysis of the impact of pathologies related to the weakness in the suspension devices.

\section{Results and Discussions}

\section{Validation with prototypical images}

Firstly, we validated our Bézier based "Material points tracking" algorithm and computation of displacements by using prototypical dynamic MR images. The captured images consist in an orange, on which a rigid body displacement in the range of $0-90 \mathrm{~mm}$ was applied. The spatial image resolution is $0.73 \mathrm{~mm}$ pixel $^{-1}$ and the size is $149 \times 261$ pixels. The illustration of validation of the automatically followed contour of the object at three instants is shown in Figure 8. For this particular case (large 
displacement between each image), each iteration of the optical flow algorithm was initialized with respect to the image barycenter to ensure the tracking of features.

The computed displacements are illustrated in Figure 9. Theoretically they should be constant along the contour and equal to 10,50 and $90 \mathrm{~mm}$. However, practically the images are discrete and are not clear everywhere. The presented results of the computed displacements are considered very close to the prescribed ones with a maximum gap of about $2 \mathrm{~mm}$. Moreover, we performed a quantitative validation of the results by evaluating the mean displacements along the contour with respect to the imposed displacements. Their standard deviation is also acceptable compared to the range of $90 \mathrm{~mm}$. The results are shown in Figure 10.

\section{Comparison and analysis of the two methods}

As mentioned above, the first method consists in the use of classic B-spline where control points can move freely, the other one is the proposed method with some features. In this part, we would like to validate our approach with the material points (features) by the comparison of the two methods. The idea is to apply the two methods on the same data set for a quantitative comparison.

The first step consisted in using the prototypical images again. However, this time the orange was rotated numerically by an angle of 0 to 20 degrees in order to illustrate the sliding problem since generally the tracking algorithms are very sensitive to the rotation. The computed angles of rotation are shown in Figure 11. Normally, the angles should be constant along the contour and equal to the imposed values. Nevertheless, it can be observed that the B-spline "Geometric contours tracking" method poorly detect the rotation. Especially, the rotation is not captured at all on the flat portion of the contour. The Bézier "Material points tracking" method better compute the rotation with few slight drifting (sliding effect) on the flat portion. As a consequence, the values calculated by the Bézier method are much closer to the imposed ones.

In order to highlight such a difference, we show a more quantitative evaluation in Figure 12. The mean angles of rotation computed by the Bézier method remain proportional to the imposed ones. Their standard deviations are less than 1 degree. On the contrary, the result given by B-spline method deviates a lot from the theoretical result.

In a second step, we also compared these two methods for a real case in the context of normal mobility. The objective is to illustrate the drifting effect and then the uncertainty of the computed displacement and shear strain. The test was run on a dynamic MR sequence of a healthy witness which contains 35 images. The spatial resolution is 1.17 mm pixel ${ }^{-1}$ and the image size is $256 \times 256$ pixels.

The visual comparison of the results given by the two methods is shown in Figure 13. The displacements and shear at the middle of the 35 images (the 20th image in the sequence) are used to highlight the difference and the drifting effect. Concerning the result given by the B-spline method, some displacements towards the end of vagina are not reasonable and ill-correlated (see the red arrows towards the bottom of Figure 13(B)). And the shear is irregular, so we do not use the measure of the shear in this part.

From the physio-anatomical point of view, some structures such as the fascias exist between bladder and vagina. The bladder, vagina and fascias are attached to each other. Such material is not supposed to stretch considerably without constraint even during the deformation, especially in the normal mobility context. So that the organ walls should neither move freely. Hence the abnormal discontinuity of the displacements on the anterior vaginal wall seems unphysical (Figure 13(B)). The same observation can be found on the posterior vaginal wall. Similarly, in Figure 13(D,E), the difference of the shear strain given by the two methods can be observed. Especially on the posterior vaginal wall, the computed shear strain in Figure 13(E) is irregular and unphysical.

The quantitative comparison is shown in Figure 14. As proved in [7], the zone around the pubis and rectosacral attachment is the low mobility area. From the point of view of anatomy, the bladder and the rectum are attached to these bones during the 

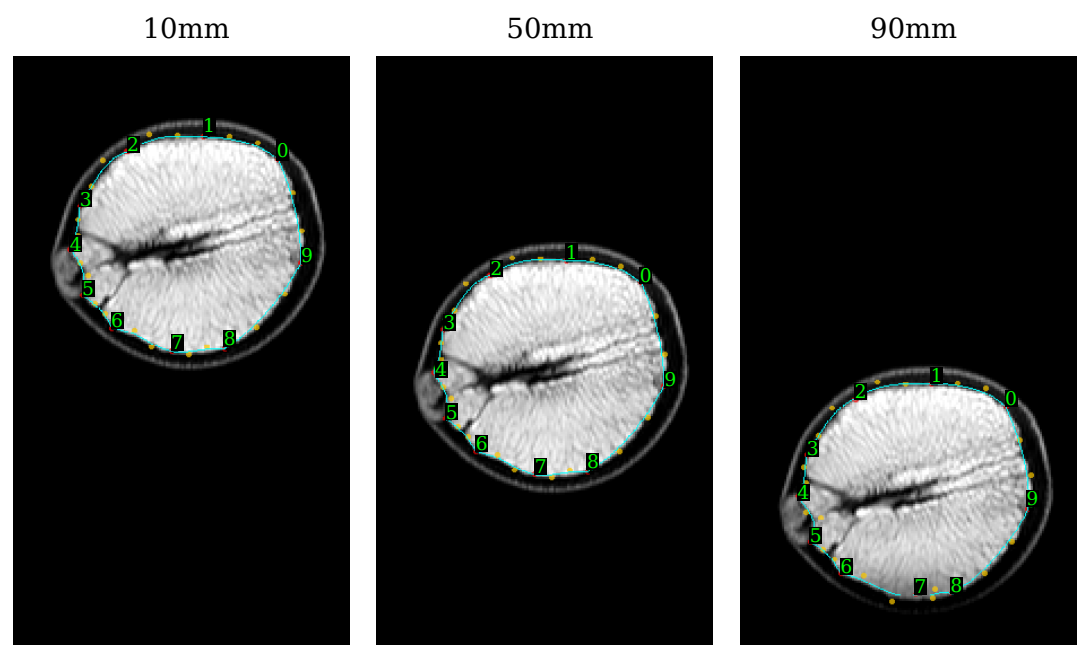

Figure 8: Validation of tracking algorithm: contour followed at the instants which corresponds to the imposed displacement of 10 , 50 and $90 \mathrm{~mm}$.

Imposed Displacement
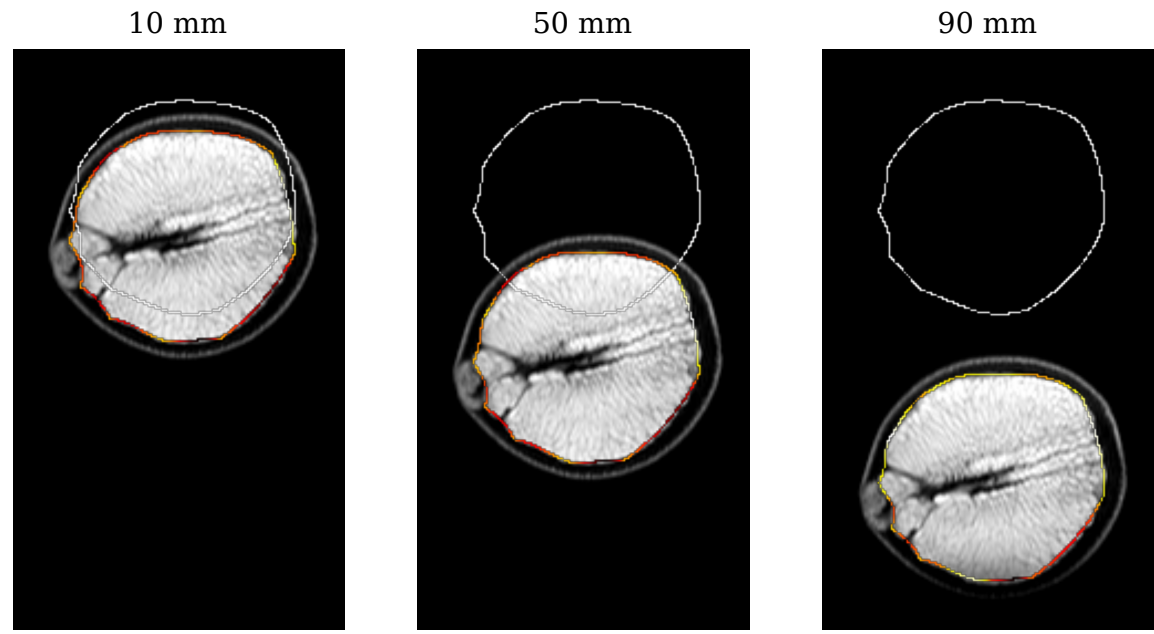

Computed displacement norm (mm)
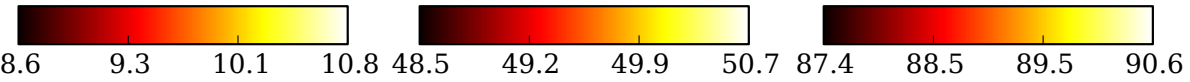

Figure 9: Validation of the displacement computation: initial contours are shown in white and the computed displacements along the contours are illustrated (the imposed displacements are 10,50 and $90 \mathrm{~mm}$ ).

deformation over time. The two methods give similar results. However, with the B-spline method, we predefined a fix point for each organ as the beginning and ending point (the same point) of the 

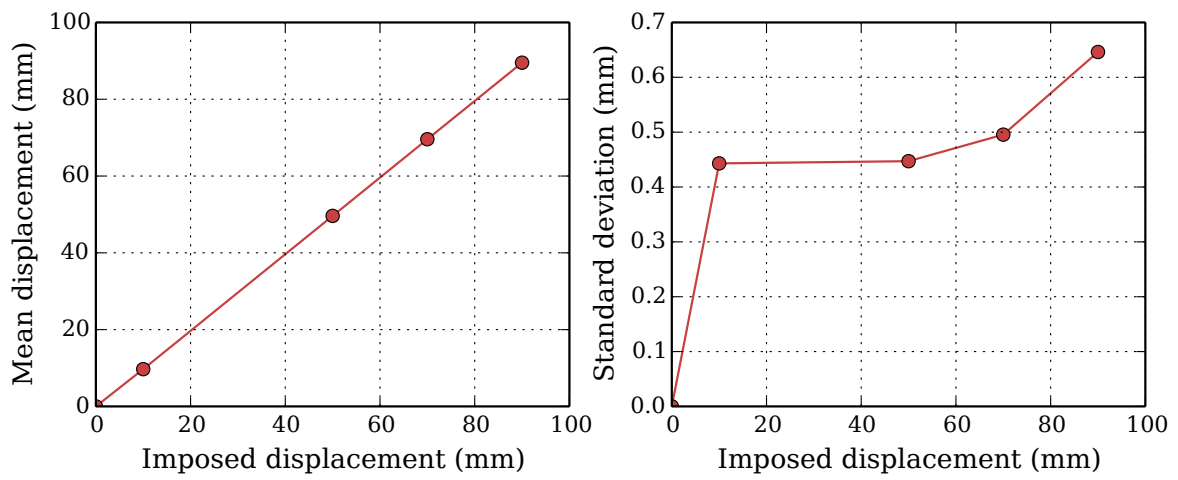

Figure 10: Quantitative validation of the displacement computation: the mean displacement along the contour and its standard deviation with respect to the imposed displacements.

B-spline to position the initial contours. Hence it is normal for the B-spline method to find these low mobility areas. On the anterior and posterior vaginal wall (zone between the vagina and the rectum) and the lower part of the rectum, high mobility can be observed, where the displacements given by the Bézier method attain a magnitude of around $22 \mathrm{~mm}$. Compared to the results in [7], the displacements are still a bit larger on the anterior vaginal wall. However, some artifacts appear along the left side of the rectum. They are principally due to the limitation of optical flow algorithm. The presence of low curvature in several areas can create artifacts on the tracking of points (see Figure 20 for illustrations). Besides, cumulative effect of errors over time can also create artifacts. because some points are not situated exactly on the contours. The main effort should be made to improve the precision of optical flow algorithm, which is to be one of the perspectives. Through all these comparisons between the results given by the B-spline "Geometric contours tracking" method, Bézier "Material points tracking" method and [7], we are in favour of the Bézier method because the tracking of material points is essential and it is more physical. In the next section, we will apply the Bézier method to more clinical cases in the context of hypermobility and hypomobility.

\section{Application to other mobility cases}

We performed the same Bézier registration method on a dynamic MRI sequence of a patient with pelvic prolapse. Because of large displacements in the context of hypermobility, the algorithm had some limitations especially when the uterus is too large. We would also mention other limitations due to unexpected effect in the image: the injected gel can flow through the cavity while the patient is pushing and some internal material of the organs can blur the image. All these effects can make the contours deviate from the truly anatomical contours. The restricted sequence from the beginning to the middle of straining contains 14 images, the spatial resolution of which is $1.15 \mathrm{~mm}$ pixel ${ }^{-1}$ and the size is $240 \times 240$ pixels. It can be observed that in the high mobility area (posterior vagina) the displacements (Figure 15B) are higher than in the normal case (Figure 14C). The magnitude of displacement is close to $25 \mathrm{~mm}$ compared to $15 \mathrm{~mm}$ for physiology.

The same process was applied on a data set of patient with pelvic endometriosis. The sequence contains 32 images of $256 \times 256$ pixels and the spatial resolution is $1.17 \mathrm{~mm} \mathrm{pixel}^{-1}$. Due to the presence of wrinkles of the rectum in the image, only the inferior part of the rectum was taken into account for the analysis. In the high mobility area, the magnitude of displacement attained around $10 \mathrm{~mm}$ even 
Bézier "Material points tracking" method

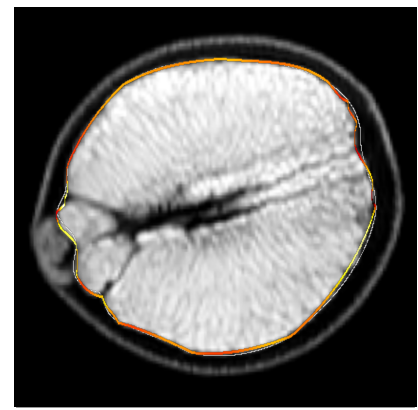

Computed rotation (degree)

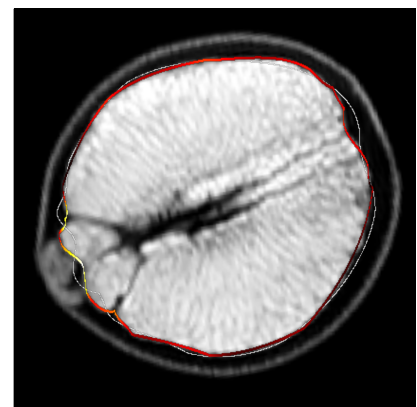

Computed rotation (degree)

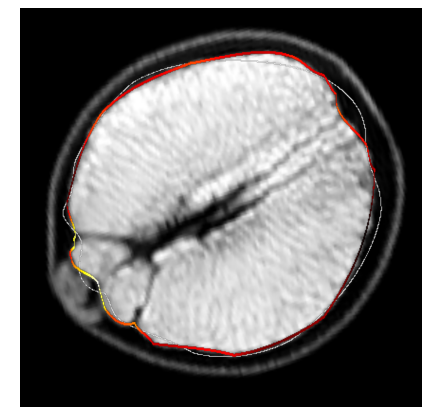

Computed rotation (degree)

\section{2}

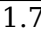

$\begin{array}{lll}2.6 & 3.0 & 8.3\end{array}$
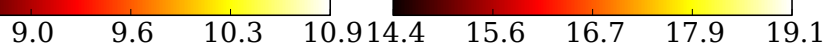

B-spline "Geometric contours tracking" method

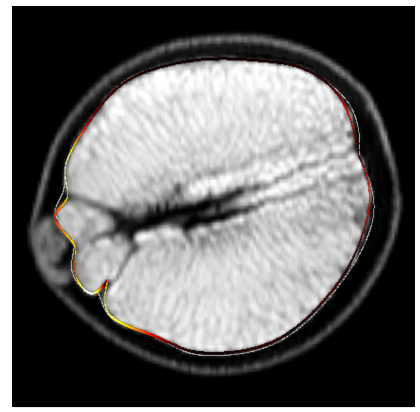

Computed rotation (degree)

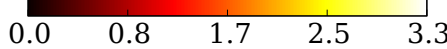

Imposed rotation (degree)

2.5

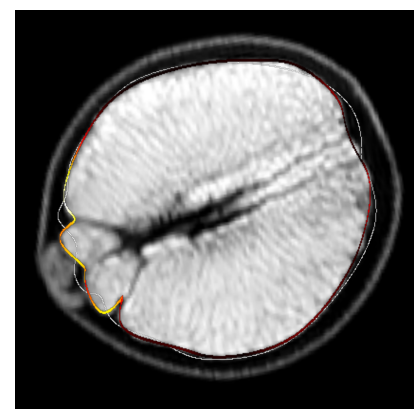

Computed rotation (degree)

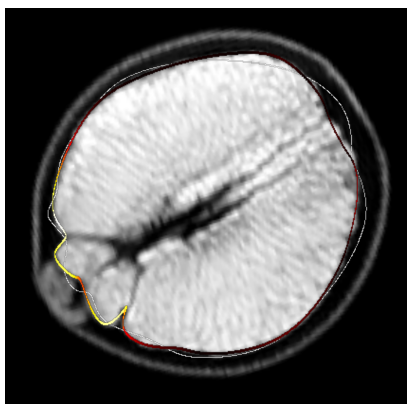

Computed rotation (degree)

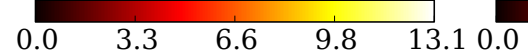

10

17.5

Figure 11: Comparison of the angles of rotation computed by two methods: the results given by Bézier "Material points tracking" method are shown on the top and the ones given by B-spline "Geometric contours tracking" method are on the bottom. The white contour is the initial one. The imposed rotations are 2.5, 10.0 and 17.5 degrees. The color map illustrate the variation along the contour in two cases.

at the end of straining, which corresponds to the context of hypomobility: the endometriosis lesions create adhesions between the organs which restrict the mobility. These examples were done on a quadcore desktop computer running at $3.10 \mathrm{GHz}$, with 16GB memory. The computational time was about $45 \mathrm{~s}$ for one image.

\section{Illustration of the shear strain}

Figure 17 shows the estimation of shear strain in the inter-organs area: anterior vagina (between bladder and vagina) and posterior vagina (between vagina and rectum). The quantification of shear is represented in a spatial-temporal shear map. In order to present more clearly the evolution of shear strain at different positions over time, the value of shear strain is presented by the change of angles (see $\frac{\pi}{2}-\alpha_{i}^{t}$ in Equation 13). During the body movement, the 
Multiorgan Motion Tracking in Dynamic MRI : Z.Jiang et al.
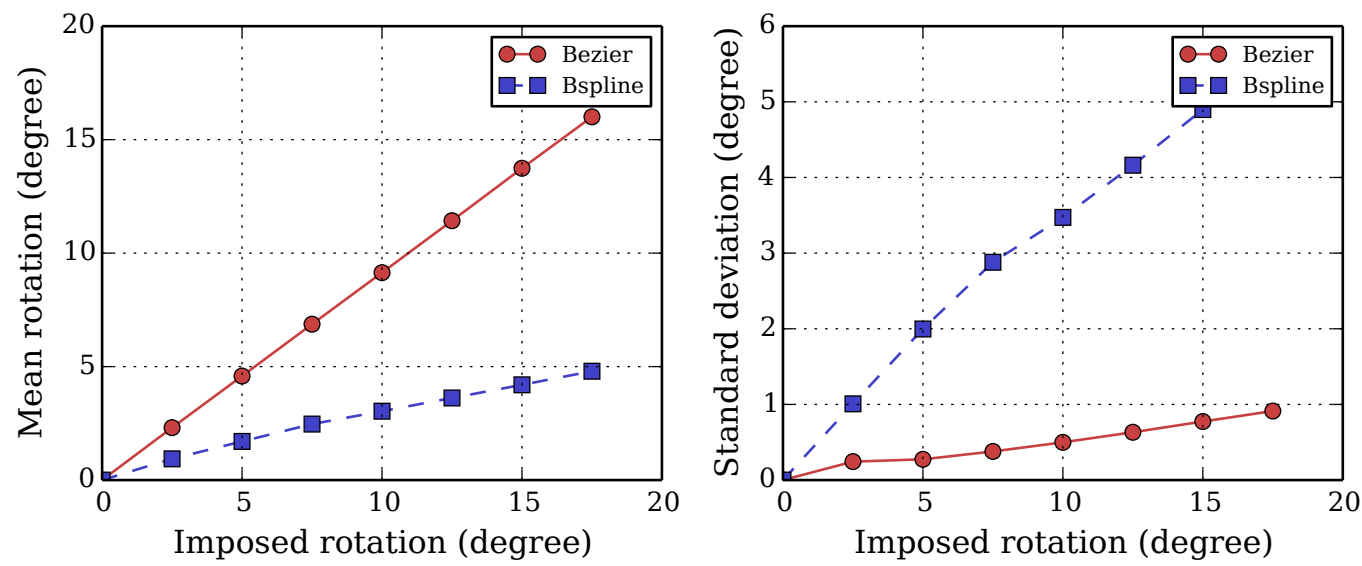

Figure 12: Quantitative comparison of the rotation computation: the mean angle of rotation along the contour and its standard deviation with respect to the imposed rotation.

curvilinear coordinate (from 0 to 20 ) represents the position on the vaginal wall (from top to bottom). The position is defined by the user by choosing a zone of interest. We used the percentage in length to indicate where the zone of interest is located on the vaginal wall. On the left-hand side figure (anterior vagina), negative values mean that the vagina descends faster than the bladder. Analogously, on the right-hand side figure (posterior vagina), negative values mean that the rectum descends faster than the vagina. It can be observed that the shear on the anterior vagina appears less important than on the posterior vagina. For the posterior vagina, visually we can observe in the dynamic MRI that the rectum descends largely under pressure. Moreover, the value of the shear is higher at the top part of the vaginal wall. This method shows indeed the spatial-temporal evolution of the shear strain, related to the invisible structures on the MR images, which cannot be visualized or quantified otherwise.

The evaluation of shear strain inter-organ can provide a more precise diagnosis and can help localize possible lesion areas before the surgery. In Figure 17-19, the estimation of shear strain is shown for the context of normal mobility, hypo-mobility and hyper-mobility respectively. It can be observed in Figure 18 that, in the case of pelvic endometriosis, the shear between the organs remains lower over time, compared to the physiological case (normal mobility). Figure 19 demonstrates a case of cystocele (occurs when the bladder pushes through to the vagina). The results need more analysis in the clinical context. However, we would like to mention a typical artifact presented on the anterior vagina in Figure 19. The discontinuity and irregularity of shear strain are due to the limitation of the optical flow algorithm that we applied. In Figure 20, it can be observed that the feature points 2,3 and 4 on the posterior bladder are not well follow in the vertical direction. This effect creates motion artifacts which produce unphysical shear strain between the bladder and the vagina.

\section{Conclusion and Perspectives}

In this paper, we present a deformable registration approach based on analytical models for multi-organ motion tracking in dynamic MRI sequences. The measured motion is then used for the evaluation of the mobility of pelvic system. The organ was defined by an analytical and parametric model, a composite Bézier curve joined at features (considered as 
A Bézier "Material points tracking"

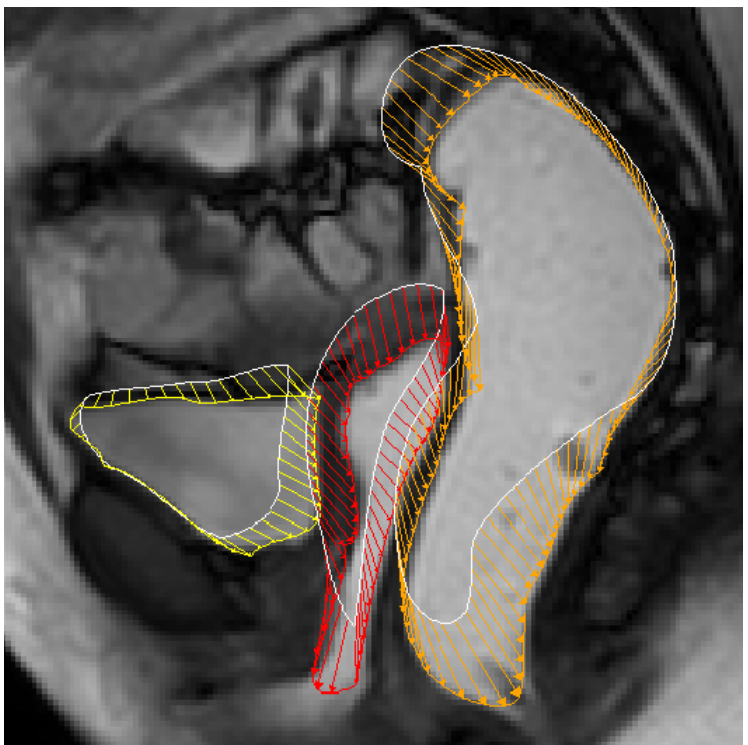

C Initial shear

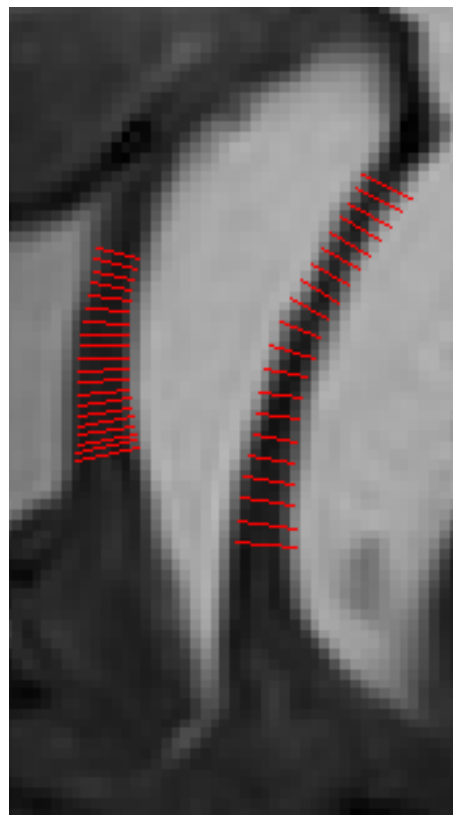

$\mathrm{D}$
B B-spline "Geometric contours tracking"
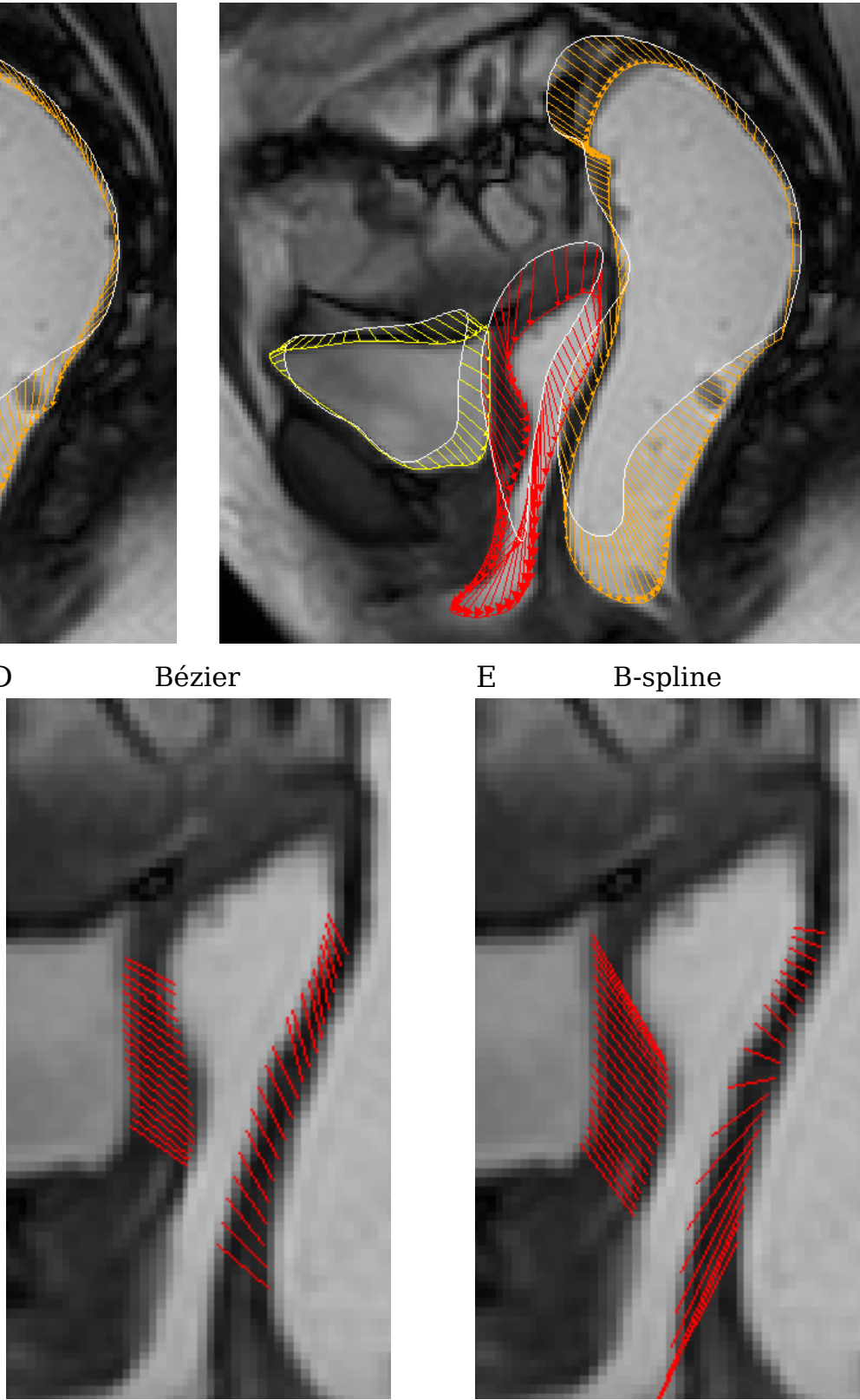

$\mathrm{E}$

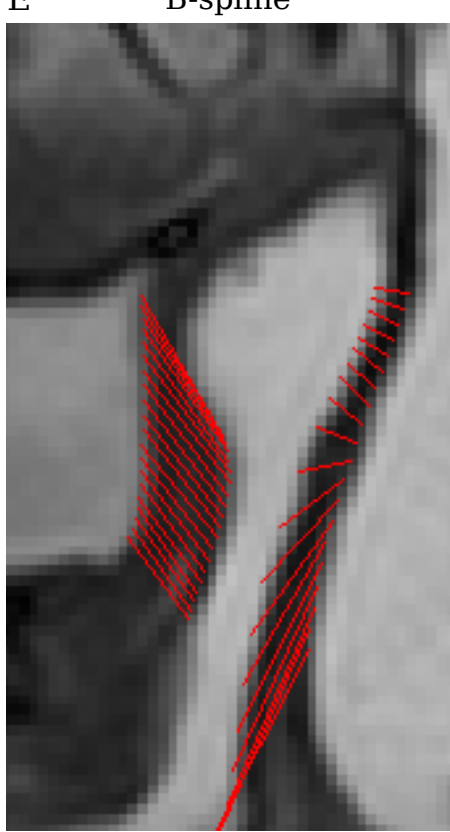

Figure 13: Illustration of visual comparison between two method. On the top: displacements at the middle of straining computed by (A) Bézier "Material points tracking" method and (B) B-spline "Geometric contours tracking" method. The white contours are the initial ones. On the bottom: (C) initial position of segments illustrating the absence of shear, and deviation of the segments at the middle of straining computed by (D) Bézier "Material points tracking" method and (E) B-spline "Geometric contours tracking" method. 

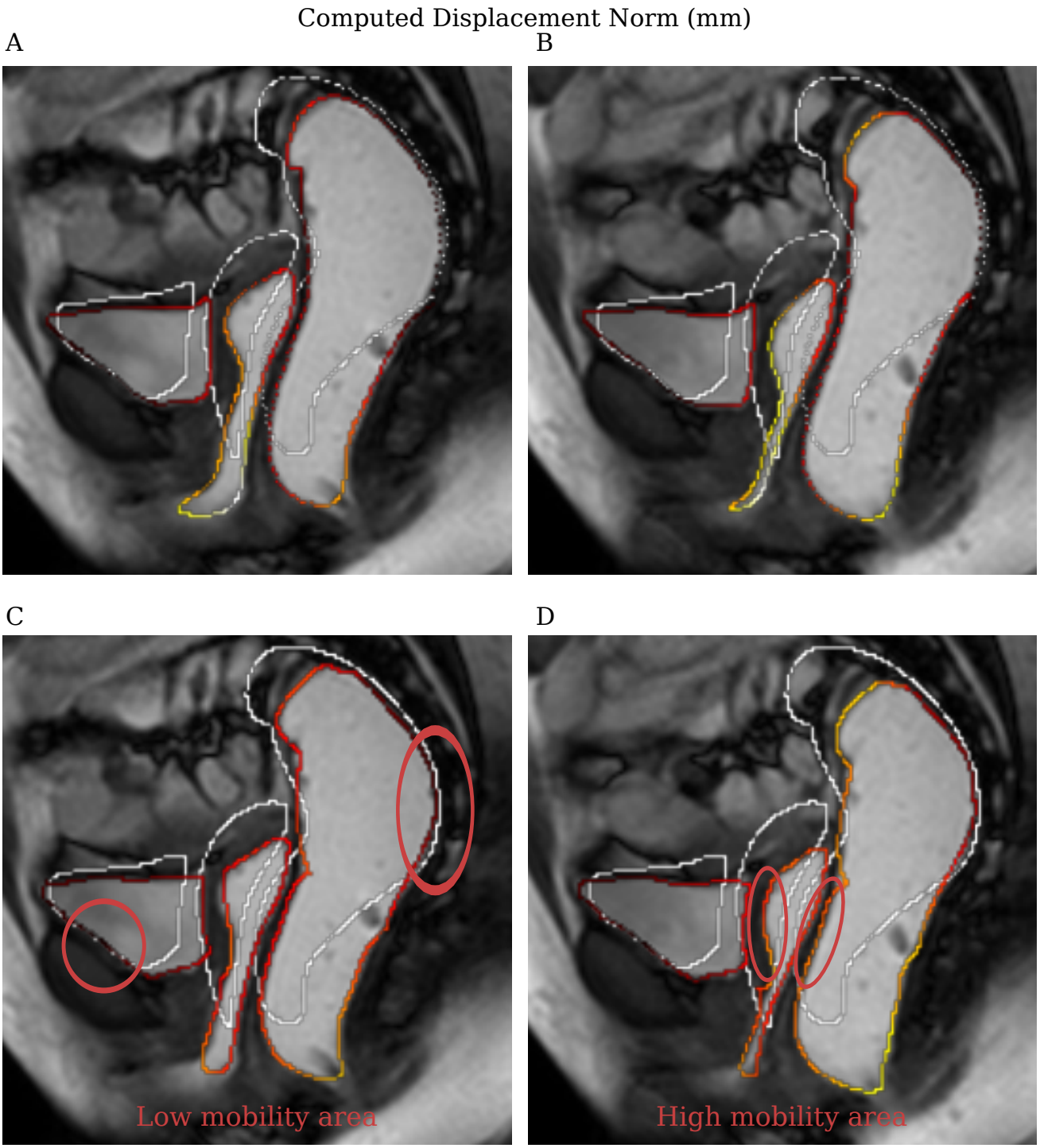

$\mathrm{D}$
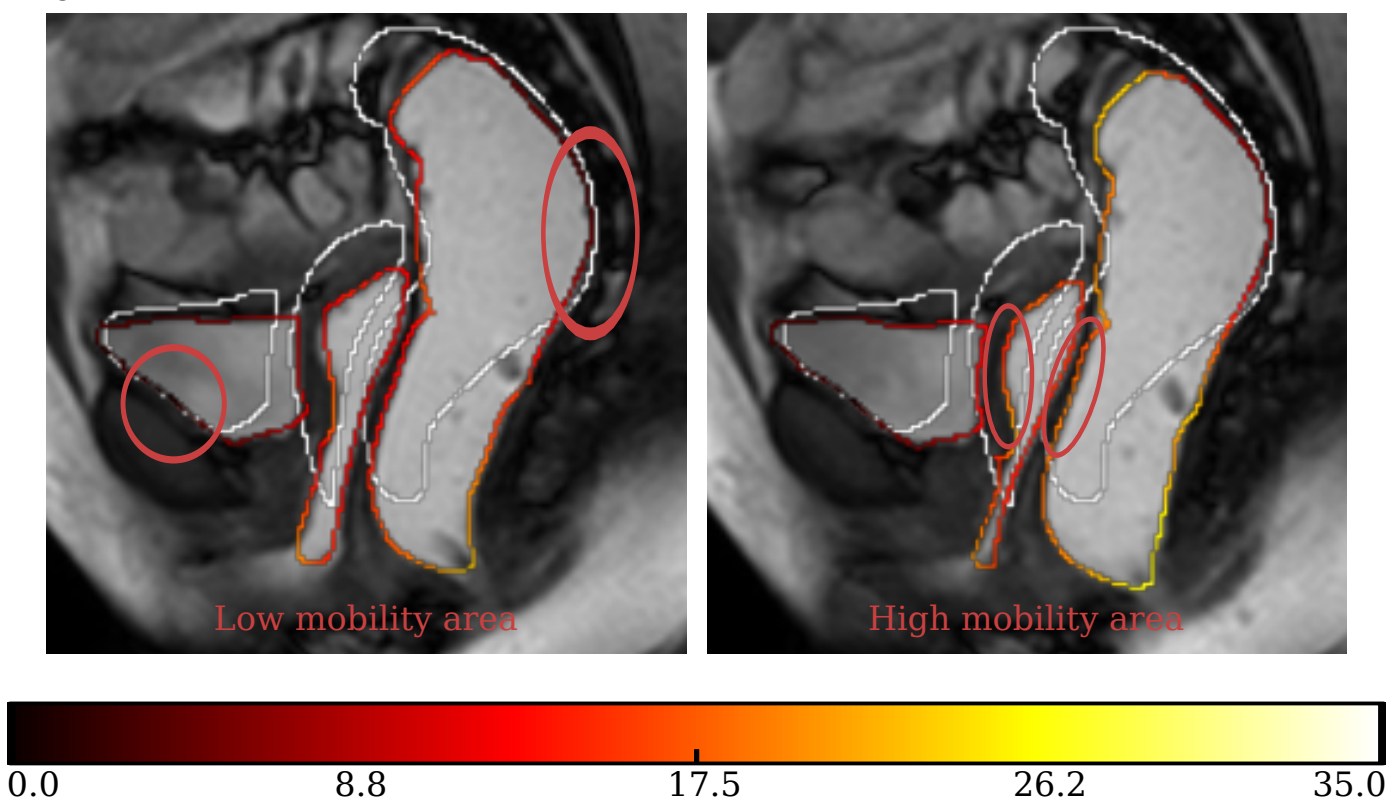

Figure 14: Quantitative comparison of the displacements computed by two methods: $(A, B)$ Displacement computed by B-spline "Geometric contours tracking" method at the middle and end of straining, (C,D) Displacement computed by Bézier "Material points tracking" method at the middle and end of straining.

materiel points). This definition can ensure better the plausibility of the measured motion. The model was then deformed to fit the organ in the dynamic image sequences by using the optical flow method 
and the virtual image correlation. Finally, thanks to the analytical formulation, we computed the displacement and shear strain field of organs during motion. Hence, the developed numerical tool, linked with the B-spline based detection, can provide a quantitative evaluation of organ motion for patientspecific cases.

The quantification of the shear strain could be used to analyse the abnormal pelvic mobility and to localize the pathological areas related to the suspension devices which can hardly be observed on medical images. The evaluation would provide information on lesions areas and their extension in case of pelvic endometriosis. The first perspective of this work is to validate the link between the measurement and the clinical usage. Such information on the structures responsible for pathologies can allow to prepare a more personalized and adapted surgery and to better inform the patient.

Secondly, concerning the contours tracking, several artifacts appeared, due to the limitation of the optical flow algorithm and unwanted effect in the images. Hence, we would also like to improve the quality and robustness of the tracking algorithm by means of determining more precisely the variables involved in the optimization, specifying the step where manual work is necessary and proposing a more integrated method. Besides, the parallel computing can be used to improve the computation time.

Thirdly, since the hypothesis of $2 \mathrm{D}$ movements in the sagittal plane is an approximation. Although we have taken maximum precautions to compute the $2 \mathrm{D}$ tangential information, another approach is to evaluate the impact of this approximation with $3 \mathrm{D}$ models. We have been carrying out a method for the registration of 3D Finite Elements models with contours of organs in a dynamic MRI sequence. The physics-based models will remedy the artifacts of contours tracking and provide full 3D movements.

Finally, as many studies have been developed on Finite Elements simulation to help understanding the physio-pathology, these experimental results would provide useful data for tests and validations in comparison with the FE simulation results. For example, the in vivo mechanical properties identifi- cation for the patient-specific medical simulation.

\section{References}

1. E. C. Samuelsson, F. T. Victor, G. Tibblin, and K. F. Svärdsudd. Signs of genital prolapse in a swedish population of women 20 to 59 years of age and possible related factors. American Journal of Obstetrics and Gynecology, 180(2):299-305, 1999.

2. S. E. Swift. The distribution of pelvic organ support in a population of female subjects seen for routine gynecologic health care. American Journal of Obstetrics and Gynecology, 183(2):277-285, 2000.

3. B. Eskenazi and M. L. Warner. Epidemiology of endometriosis. Obstet. Gynecol. Clin. North Am., 24(2):235-258, 1997.

4. C. Meuleman, B. Vandenabeele, S. Fieuws, C. Spiessens, D. Timmerman, and T. D'Hooghe. High prevalence of endometriosis in infertile women with normal ovulation and normospermic partners. Fertil Steril., 92(1):68-74, 2009.

5. R. C. Bump, A. Mattiasson, K. Bø, L. P. Brubaker, J. O. DeLancey, P. Klarskov, B. L. Shull, and A. R. Smith. The standardization of terminology of female pelvic organ prolapse and pelvic floor dysfunction. American Journal of Obstetrics and Gynecology, 175(1):10-17, 1996.

6. M. Dell'oro, P. Collinet, G. Robin, and C. Rubod. Multidisciplinary approach for deep endometriosis: interests and organization. Gynecol. Obstet. Fertil., 41(1):58-64, 2013.

7. P. Lecomte-Grosbras, J.-F. Witz, M. Brieu, N. Faye, M. Cosson, and C. Rubod. Quantification of pelvic mobility on dynamic magnetic resonance images: Using mechanical insight to help diagnose pelvic pathologies. Strain., 51(4):301-310, 2015.

8. H. K. Pannu, H. S. Kaufman, G. W. Cundiff, R. Genadry, D. A. Bluemke, and E. K. Fishman. Dynamic MR imaging of pelvic organ prolapse: Spectrum of abnormalities 1 . Radiographics, 20(6):1567-1582, 2000.

9. G. V. Rao, C. Rubod, M. Brieu, N. Bhatnagar, and M. Cosson. Experiments and finite element modelling for the study of prolapse in the pelvic floor system. Computer Methods in Biomechanics and Biomedical Engineering, 13(3):349-357, 2010.

10. A. Vallet, J.-F. Witz, C. Rubod, M. Brieu, and M. Cosson. Simulation of pelvic mobility: topology optimization of ligamentous system. Computational Methods in Biomechanics and Biomedical Engineering, 14(1):159-163, 2011.

11. P. Lecomte-Grosbras, M. Nassirou-Diallo, J.-F. Witz, D. Marchal, J. Dequidt, S. Cotin, M. Cosson, C. Duriez, and $\mathrm{M}$. Brieu. Towards a better understanding of pelvic system disorders using numerical simulation. In MICCAI, Nagoya, Japan, 2013.

12. D. L. G. Hill, P. G. Batchelor, M. Holden, and D. J. Hawkes. Medical image registration. Physics in medicine and biology, 46(3):R1-45, 2001.

13. S. Klein, M. Staring, K. Murphy, M. A. Viergever, and J. P. W. Pluim. elastix: a toolbox for intensity based medical 
Multiorgan Motion Tracking in Dynamic MRI : Z.Jiang et al.

image registration. IEEE Transactions on Medical Imaging., 29(1):196-205, 2010.

14. D. P. Shamonin, E. E. Bron, B. P. F. Lelieveldt, M. Smits, S. Klein, and M. Staring. Fast parallel image registration on cpu and gpu for diagnostic classification of alzheimer's disease. Frontiers in Neuroinformatics., 7(50):1-15, 2014.

15. F. P. Oliveira and J. M. Tavares. Medical image registration: a review. Computer Methods in Biomechanics and Biomedical Engineering., 17(2):73-93, 2014.

16. D. Lecompte, A. Smits, S. Bossuyt, H. Sol, J. Vantomme, D. Van Hemelrijck, and A. M. Habraken. Quality assessment of speckle patterns for digital image correlation. Optics and Lasers in Engineering., 44(11):1132-1145, 2006.

17. N. F. Osman, E. R. McVeigh, and J. L. Prince. Imaging heart motion using harmonic phase mri. IEEE Trans Med Imaging., 19(3):186-202, 2000.

18. J. Lee, J. Woo, F. Xing, E. Z. Murano, M. Stone, and J. L. Prince. Semi-automatic segmentation for $3 d$ motion analysis of the tongue with dynamic mri. Comput Med Imaging Graph., 38(8):714-724, 2014.

19. Z. Jiang, J.-F. Witz, P. Lecomte-Grosbras, J. Dequidt, C. Duriez, M. Cosson, S. Cotin, and M. Brieu. B-spline based multi-organ detection in magnetic resonance imaging. Strain., 51(3):235-247, 2015.

20. F. F. Berendsen, A. N. T. J. Kotte, M. A. Viergever, and J. P. W. Pluim. Registration of organs with sliding interfaces and changing topologies. In Proc. SPIE, San Diego, California, USA, volume 9034, pages 90340E-90340E-7, 2014.

21. B. Hamm and R. Forstner, editors. Diagnostic Imaging MRI and CT of the Female Pelvis. Diagnostic Imaging. Springer-Verlag Berlin Heidelberg, 2007.

22. L. Piegl and W. Tiller. The NURBS Book (2nd Edition). Monographs in visual communication, SpringerVerlag Berlin Heidelberg, 1997.

23. B. Semin, H. Auradou, and M. L. M. François. Accurate measurement of curvilinear shapes by virtual image correlation. European Physical Journal: Applied Physics, 56(1):10701-p1-10, 2011.

24. J. Réthoré, T. Elguedj, P. Simon, and M. Coret. On the use of nurbs functions for displacement derivatives measurement by digital image correlation. Experimental Mechanics, 50(7):1099-1116, 2010.

25. J. Réthoré and M. L. M. François. Curve and boundaries measurement using b-splines and virtual images. Optics and Lasers in Engineering, 52:145-155, 2014.

26. B. D. Lucas and T. Kanade. An iterative image registration technique with an application to stereo vision. In IJCAI, volume 81, pages 674-679, 1981.

27. T. Brox and J. Malik. Large displacement optical flow: descriptor matching in variational motion estimation. IEEE Transactions on Pattern Analysis and Machine Intelligence, 33(3):500-513, 2011.

(C) 2017 The Authors - Journal: Strain 


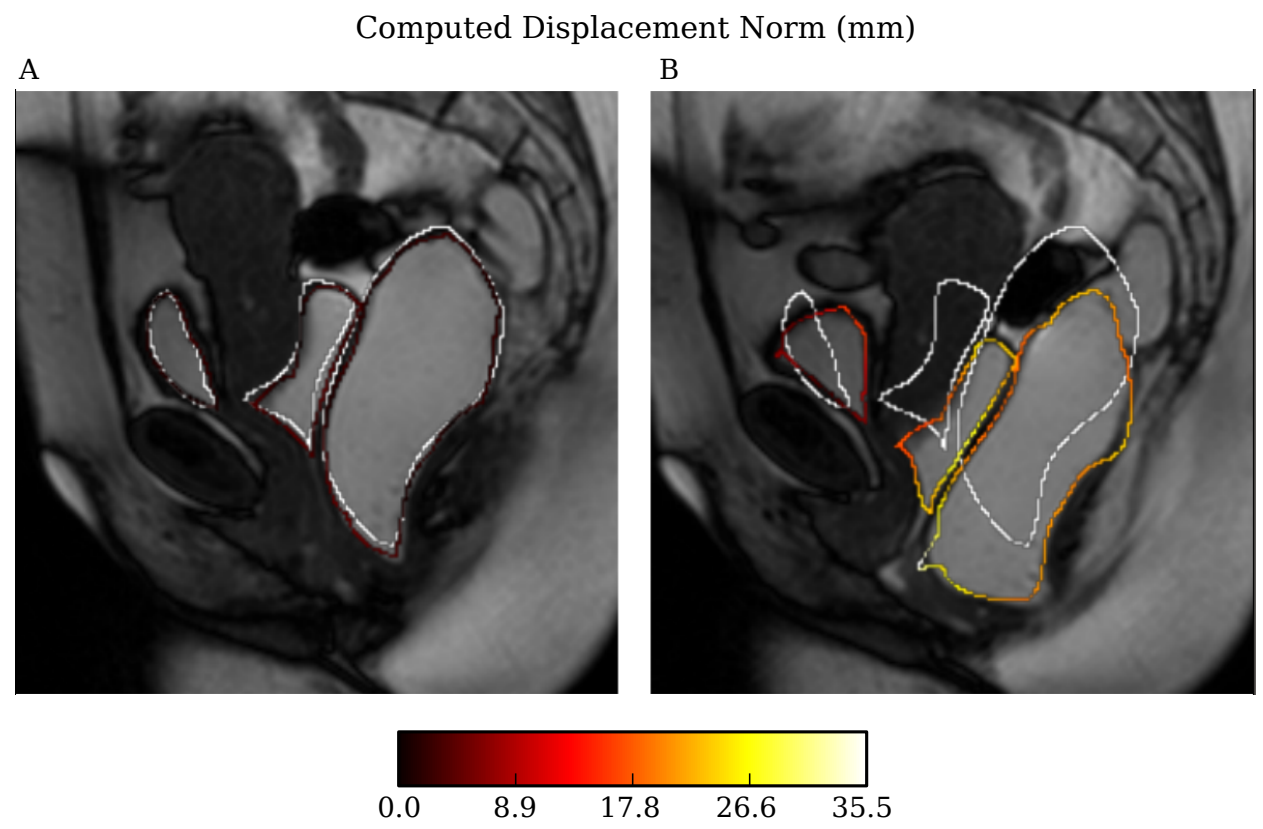

Figure 15: Quantitative evaluation of the displacement field in the context of hypermobility: (A) Displacements at the beginning of straining and (B) at the middle of straining. The white contours are the initial ones.

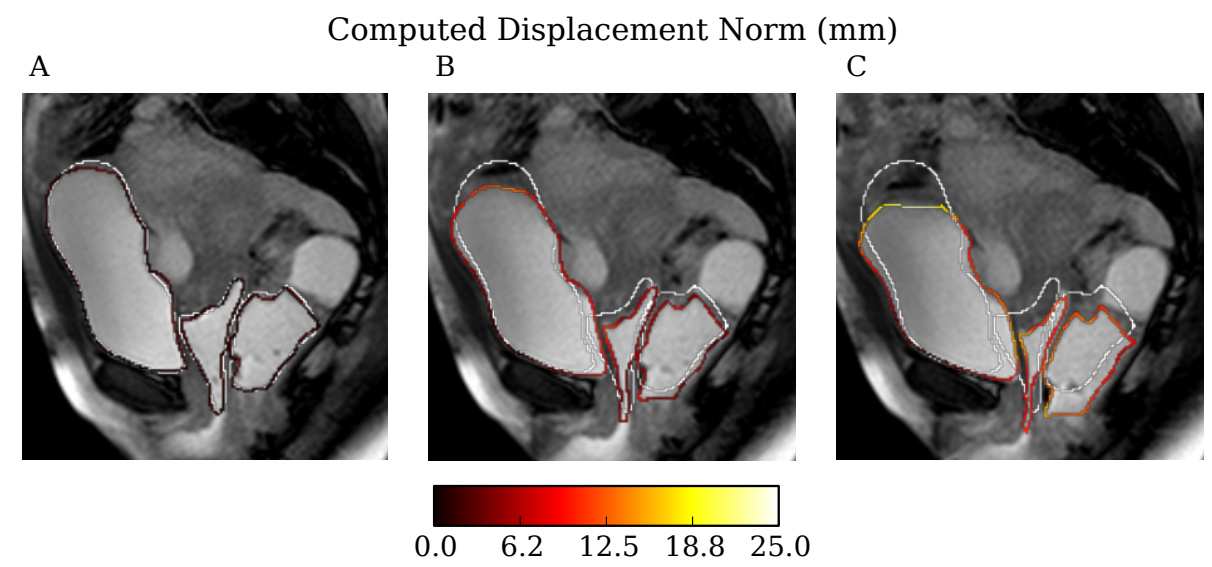

Figure 16: Quantitative evaluation of the displacement field in the context of hypomobility: (A) Displacements at the beginning of straining, $(B)$ at the middle of straining and $(C)$ at the end of straining. The white contours are the initial ones. 
Position Anterior Vagina

$33.7 \%=l_{1} /\left(l_{1}+l_{2}+l_{3}\right)$

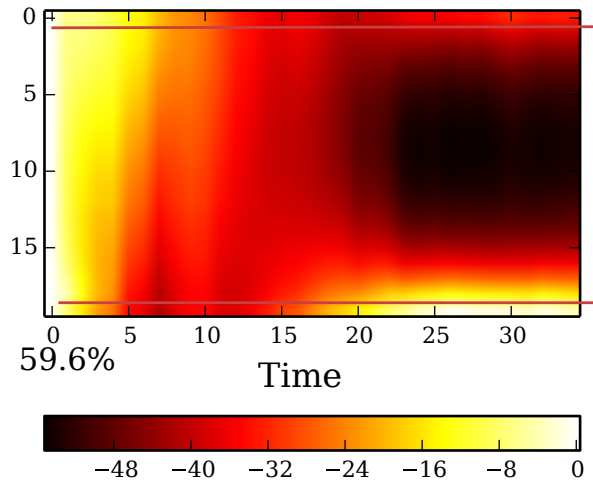

Shear estimation (degree)
Position Posterior Vagina

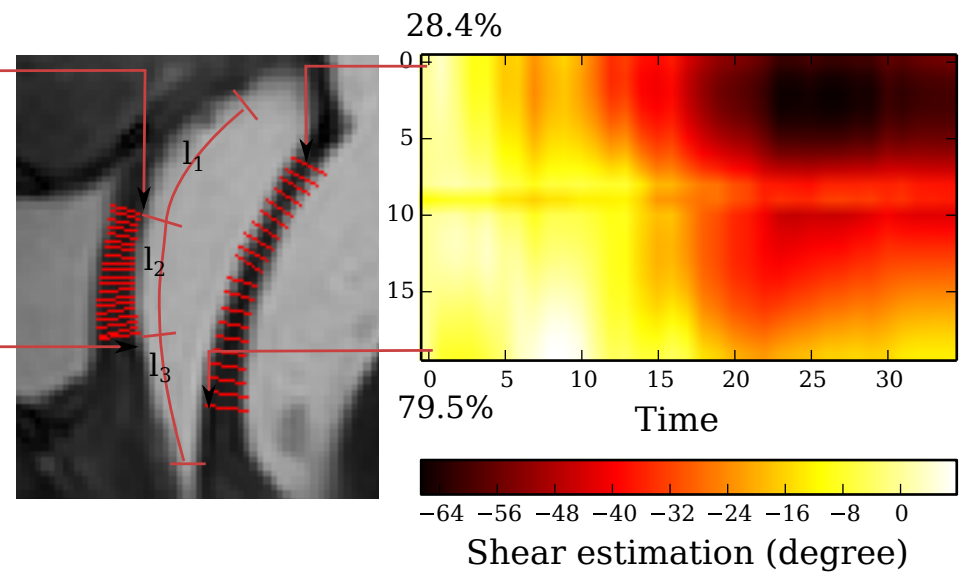

Figure 17: Spatial and temporal shear strain map from the beginning to the end of straining, in the context of normal mobility.

\section{Position Anterior Vagina}

$28.8 \%$

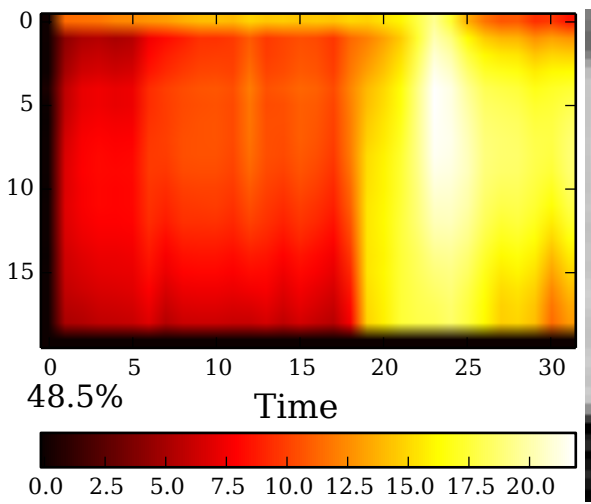

Shear estimation (degree)
Position Posterior Vagina

$37.3 \%$
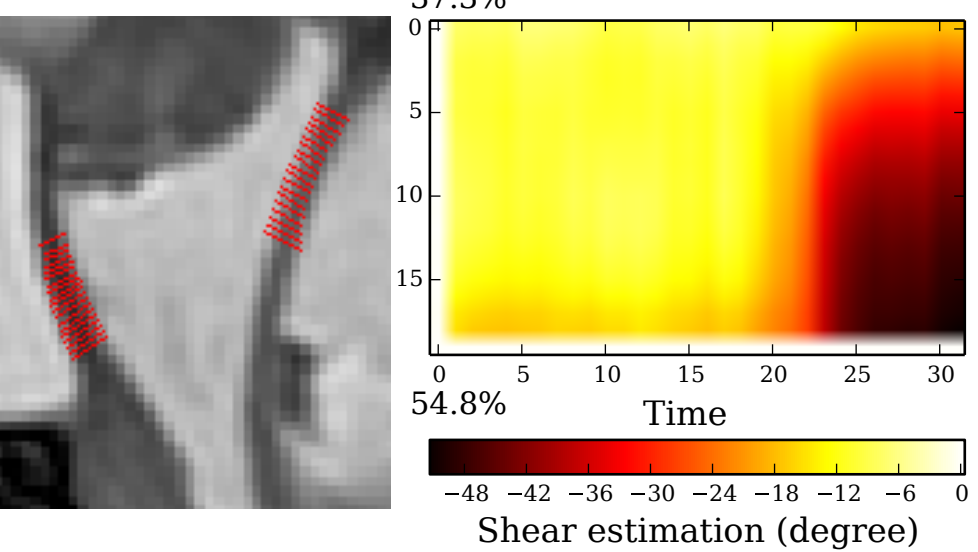

Figure 18: Spatial and temporal shear strain map from the beginning to the end of straining, in the context of hypo-mobility. 
Position Anterior Vagina

$28.1 \%$
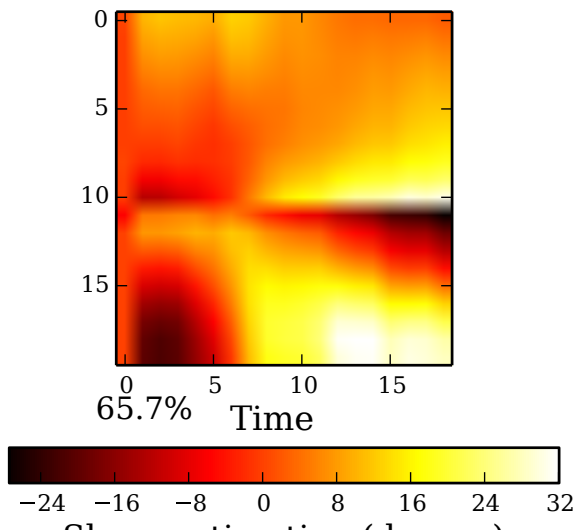

Shear estimation (degree)
Position Posterior Vagina

$27.0 \%$
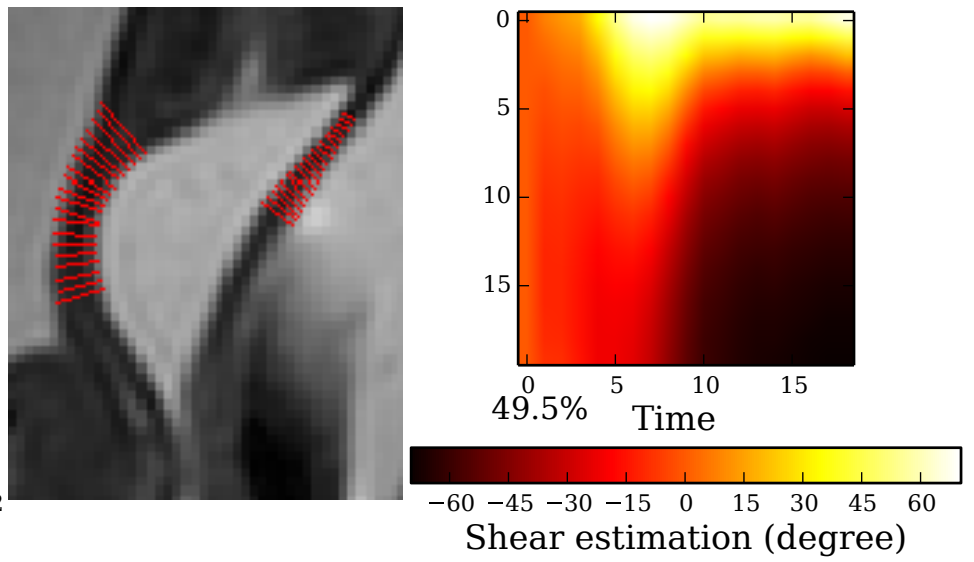

Figure 19: Spatial and temporal shear strain map from the beginning to the end of straining, in the context of hyper-mobility.
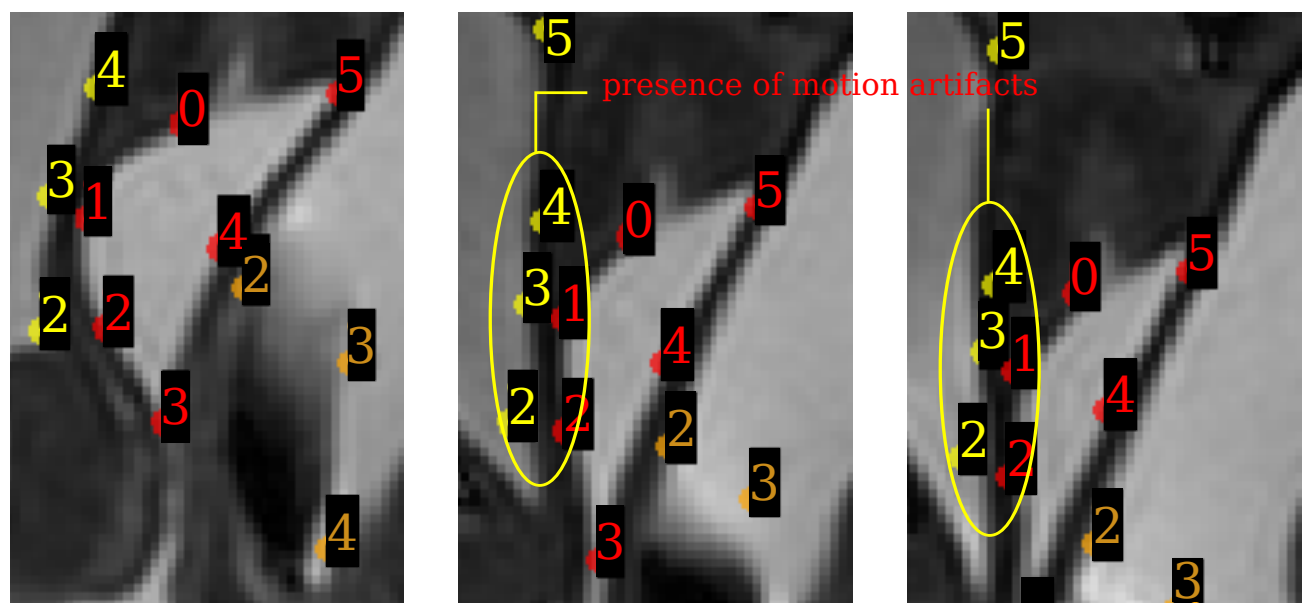

Figure 20: Illustration of typical motion artifacts in the context of hyper-mobility, due to the limitations of optical flow algorithm: On the posterior of the bladder, feature point 2,3 and 4 are not well followed in the vertical direction. Such effect can create unwanted shear strain. 